\title{
Mit Herz und Verstand: Rolle der internen Kommunikation für die Etablierung neuer Nachhaltigkeitsstrategien
}

\author{
Lea Strottner ${ }^{1} \cdot$ Simone Huck-Sandhu \\ Eingegangen: 7. August 2020 / Überarbeitet: 7. Juli 2021 / Angenommen: 16. Dezember 2021 / Online publiziert: 18. Januar 2022 \\ (c) Der/die Autor(en) 2022
}

\section{Zusammenfassung}

Die Erwartungen vieler Stakeholder an unternehmerische Nachhaltigkeit und Verantwortung sind gestiegen. In der Folge begeben sich Unternehmen in einen Transformationsprozess und verankern Nachhaltigkeitsziele und -strategie in ihrer Unternehmensstrategie. Dies bringt in der Regel einen fundamentalen Veränderungsprozess für die Mitarbeitenden mit sich. Die neue Nachhaltigkeitsorientierung intern durch- und umzusetzen bedeutet u.a., dass die interne Veränderungskommunikation die Nachhaltigkeitsthematik aktiv zu vermitteln und den Veränderungsprozess kommunikativ zu begleiten hat. Ziel dieses Beitrags ist es, Grundlagen, Rahmenbedingungen und Erfolgsfaktoren der internen Veränderungskommunikation im Zusammenhang mit der Etablierung einer Nachhaltigkeitsstrategie im Unternehmen zu analysieren und deren kommunikative Implementierung und Institutionalisierung zu beleuchten. Im Rahmen einer qualitativen Studie wurden Leitfadengespräche mit Verantwortlichen für interne Nachhaltigkeitskommunikation in Großunternehmen geführt. Die Ergebnisse bestätigen, dass interne Kommunikation unter bestimmten Rahmenbedingungen Treiber für eine neue, nachhaltigkeitsorientiere Unternehmensstrategie und die damit einhergehenden Wandlungsprozesse sein kann. Es deuten sich aber auch Besonderheiten an, die - anders als in allgemeinen Veränderungsprozessen - bei der Etablierung von Nachhaltigkeitsstrategien qualitativ wichtiger zu sein scheinen. Um die neue Strategie kognitiv und affektiv in den Köpfen und Herzen der Mitarbeitenden zu verankern, bedarf es einer systematischen Veränderungskommunikation, die über Werte, Kultur und kommunikatives Sensegiving Nachhaltigkeit verankert. Dazu kommen spezifische Kommunikationsmodi, -formate und -medien zum Einsatz. Für die Forschung liefert der Beitrag explorative Hinweise an einer bislang kaum beleuchteten Nahtstelle von interner Veränderungs- und Nachhaltigkeitskommunikation. Für die Unternehmenspraxis identifiziert er Rahmenbedingungen und Herausforderungen und zeigt auf, wie Ziele, Aufgabe und Maßnahmen der Kommunikationsarbeit für die Etablierung neuer Nachhaltigkeitsstrategien umgesetzt werden können.

Schlüsselwörter Interne Kommunikation · Nachhaltigkeitskommunikation · Veränderungskommunikation · Nachhaltigkeitsstrategie

Simone Huck-Sandhu

simone.huck-sandhu@hs-pforzheim.de

1 Fakultät für Wirtschaft und Recht, Hochschule Pforzheim,

Pforzheim, Deutschland 


\title{
Implementing change through internal communication: facilitating sustainability strategies
}

\begin{abstract}
Stakeholders' expectations regarding corporate sustainability and responsibility have been rising in recent years. As a result, companies are embarking on a transformation process. In many cases, a fundamental change process is required to embed sustainability goals in corporate strategy. Implementing the new sustainability orientation internally means, among other things, that internal communication with employees has to actively convey sustainability goals and facilitate the change process. The aim of this article is to conceptualize internal change communication for establishing new corporate sustainability strategies. In a qualitative approach, interviews were conducted with internal communication managers from large companies in Germany. The results suggest that under specific conditions internal change communication can indeed be a driver for a new, sustainability-oriented corporate strategy and the associated processes of change. In order to implement sustainability in the 'minds and hearts' of employees change communication needs to be based on values, culture and communicative sensegiving via specific communication modes, formats and media. Regarding research, this study provides explorative findings that further studies in internal change communication and sustainability communication could build upon. For communication practitioners, the article identifies relevant conditions and challenges and names goals, tasks and instruments for facilitating employees' adoption of a new sustainability strategy.
\end{abstract}

Keywords Internal communication $\cdot$ Sustainability communication $\cdot$ Change communication $\cdot$ Sustainability strategy

\section{Einleitung}

Unternehmerische Nachhaltigkeit und Verantwortung haben sich heutzutage in vielen Unternehmen etabliert (Schaltegger et al. 2007, S. 3). Vehemente Forderungen der Stakeholder nach mehr Transparenz unternehmerischer Werte und nach einem Unternehmenshandeln, das soziale, ökologische und ökonomische Aspekte gleichberechtigt berücksichtigt, zeigen in den letzten Jahren verstärkt Wirkung. Es sind nicht mehr länger nur NGOs, Verbraucher und Politiker, die sich für das Nachhaltigkeitsprofil eines Unternehmens interessieren. Auch Investoren, Konsumenten und die Mitarbeitenden achten verstärkt auf Nachhaltigkeit.

Unternehmen, so die Erwartung, werden sich künftig mit weiter steigenden Anforderungen an unternehmerische Nachhaltigkeit und Verantwortung konfrontiert sehen (Wagner 2019, S. 26). Erforderlich ist in vielen Fällen eine grundlegende strategische Neuausrichtung, die jedoch oft einen fundamentalen Veränderungsprozess erforderlich macht (Prexl 2010, S. 109). Um die Nachhaltigkeitsorientierung in einem solchen Veränderungsprozess intern durchund umzusetzen, bedarf es neben dem Veränderungsmanagement auch einer internen Veränderungskommunikation zur Nachhaltigkeitsthematik sowie der kommunikativen Begleitung des damit verbundenen Transformationsprozesses (Krüger 2014, S. 20). Laut der European Communication Monitor-Studie, in deren Rahmen rund 2700 Kommunikationsfachleute in 46 Ländern befragt wurden, hat die Auseinandersetzung mit der nachhaltigen Unternehmensentwicklung im Rahmen der Unternehmenskommunikation seit 2018 an Bedeutung gewonnen (Zerfaß et al. 2019, S. 54). Dennoch mangelt es in der Kommunikationspraxis oftmals an einer konsequenten Integration von Nachhaltigkeit auf operativer Ebene (Wagner 2019, S. 3).

Es gilt, Herz und Verstand der Mitarbeitenden zu erreichen: Ihr Bewusstsein für unternehmerische Nachhaltigkeit, Wissensstand, Einstellungen und Verhalten sind erfolgskritisch dafür, dass ein Unternehmen eine neue Nachhaltigkeitsorientierung realisieren kann. Allerdings: Gerade Veränderungen, die an Einstellungen und gewohnten Verhaltensweisen rütteln, sind schwer zu ändern (Deutinger 2017, S. 41). Sie geben Mitarbeitenden Stabilität und Konstanz, aber müssen sich im Wandel verändern. Eine nachhaltigkeitsorientierte Unternehmensstrategie zu implementieren ist ohne die Akzeptanz und Mitwirkung von Mitarbeitenden und somit ohne interne Veränderungskommunikation kaum möglich (Wagner 2019, S. 34). Allerdings ist die Rolle, die die interne Kommunikation für die Etablierung neuer Nachhaltigkeitsstrategien spielt, bislang wenig erforscht (Mast 2008, S. 16). Die Kommunikationsforschung, insbesondere im Feld der Organisations- und Unternehmenskommunikationsforschung, beschäftigt sich mit interner Kommunikation und Veränderungskommunikation im Allgemeinen. Das Forschungsfeld der Nachhaltigkeitskommunikation legt den Fokus bislang stark auf externe Kommunikation. Darüber blendet es die spezifischen Herausforderungen, denen sich Unternehmen gerade in der internen Veränderungskommunikation gegenübersehen, bislang weitgehend aus.

Die Frage, welche Rolle und Relevanz der internen Kommunikation im strategischen Veränderungsprozess hin zur Etablierung einer neuen Nachhaltigkeitsstrategie zukommt, ist an der Nahtstelle von interner Kommunikation und Nachhaltigkeitskommunikation angesiedelt und bis dato v.a. empirisch kaum untersucht (Gentile et al. 2015): „It remains unknown what happens to CSR concepts when 
they are transformed from formal decisions at the top of the hierarchy to concrete action in the rest of the organization", konstatieren Gentile et al. (2015, S. 19). Mast (2008, S. 16) sieht die geringe Anzahl an Publikationen zum Thema u. a. darin begründet, dass Nachhaltigkeit trotz ihrer Mehrdimensionalität und Komplexität als verhältnismäßig „leicht kommunizierbar“" gilt. Allerdings weisen Veränderungsprozesse zur Etablierung von Nachhaltigkeitsstrategien Besonderheiten auf, die sie von breiter erforschten anderen Veränderungsprozessen unterscheiden und - so deutet auch die in diesem Beitrag vorgestellte qualitative Untersuchung der Kommunikationspraxis in Unternehmen an - darüber spezifische Anforderungen an interne Veränderungskommunikation stellen.

Dieser Beitrag geht der Frage nach, welche Rolle die interne Kommunikation für die Etablierung neuer Nachhaltigkeitsstrategien in Unternehmen spielen kann. Dabei müssen auch Rahmenbedingungen in den Blick genommen werden, da sie - positiv wie negativ - Treiber für die Rolle, Reichweite und Erfolgsfaktoren einer solchen Kommunikation in strategischen Veränderungsprozessen zur Etablierung einer Nachhaltigkeitsstrategie sein können. Der Fokus des Beitrags liegt auf der internen Veränderungskommunikation mit Mitarbeitenden zur Etablierung, kommunikativen Institutionalisierung und Implementierung neuer Nachhaltigkeitsstrategien. Im Rahmen einer qualitativen Studie wird anhand von Leitfadengesprächen mit Kommunikationsfachleuten aus Unternehmen explorativ analysiert, wie eine solche Kommunikation angelegt, eingesetzt und ausgestaltet werden kann, um eine nachhaltigkeitsorientierte Strategieveränderung kognitiv und affektiv bei den Mitarbeitenden zu verankern.

\section{Zum Stand der Literatur}

Für die Frage, welche Rolle interne Kommunikation bei der Etablierung neuer Nachhaltigkeitsstrategien spielt, welche Rahmenbedingungen und Erfolgsfaktoren dabei vorliegen und wie die Veränderungskommunikation gestaltet werden kann, bedarf es zunächst der theoretischen Rahmung. Zunächst wird die interne Nachhaltigkeitskommunikation als Teilbereich des internen Kommunikationsmanagements vorgestellt und in ihrer Relevanz für den Veränderungsprozess dargestellt. Mit dem genaueren Blick auf die spezifischen Ziele und Aufgaben, die ihr in Veränderungsprozessen zukommt, wird die Rolle der internen Nachhaltigkeitskommunikation für die strategische Neuausrichtung zu Nachhaltigkeitsthemen näher beleuchtet. Abschließend stehen die spezifischen Rahmenfaktoren und Herausforderungen, die bei einer nachhaltigkeitsorientierten Strategieimplementierung gelten, im Blickpunkt. Daraus werden fünf Forschungsfragen abgeleitet, die mittels strukturierter Leitfadengespräche in Kap. 3 empirisch untersucht werden.

\subsection{Interne Nachhaltigkeitskommunikation und der ,Faktor Mensch'}

Die interne Nachhaltigkeitskommunikation stellt einen Teilbereich der internen Unternehmenskommunikation dar. Organisationsinterne Kommunikation umfasst zunächst alle „kommunikativen Prozesse, die sich in einem Unternehmen zwischen dessen Mitgliedern abspielen“ (Mast 2019, S. 283). Von interner Unternehmens- oder Mitarbeiterkommunikation ist immer dann die Rede, wenn es sich um die strategisch geplante, gesteuerte Kommunikation eines Unternehmens an bzw. mit seinen Mitarbeitenden handelt (Huck-Sandhu 2020, S. 5). Interne Nachhaltigkeitskommunikation, wie sie im Rahmen dieses Beitrags untersucht wird, ist somit eine strategische Kommunikationsfunktion (Bekmeier-Feuerhahn et al. 2017, S. 90; Bolton et al. 2011, S. 61), die Mitarbeitende und Führungskräfte über die Nachhaltigkeitsstrategie informiert, es ihnen ermöglicht, sich ein Urteil über das unternehmerische Nachhaltigkeitsengagement zu bilden und die damit verbundene Werte sowie eine gemeinsame Weltanschauung vermittelt (Weder und Karmasin 2015, S. 937; Sawczyn-Müller und Krohn 2017, S. 11).

Interne Nachhaltigkeitskommunikation operiert mit einer hohen Themenkomplexität: Nachhaltigkeit als Thema ist mehrdimensional angelegt (Mast und Fiedler 2007, S. 569f.). Hinzu kommt die Herausforderung, den abteilungsübergreifenden, interdisziplinären Informationsaustausch sicherzustellen (Brugger 2010, S. 178, 208) und Widerstände zu überwinden. Entstehen zwischen dem kommunizierten Engagement und dem wahrgenommenen Handeln Diskrepanzen, schlägt sich dies negativ auf die wahrgenommene Integrität, Rechtschaffenheit und Vertrauenswürdigkeit sowie, in der Folge, auf die Glaubwürdigkeit und das Unternehmensimage nieder (Kleinfeld und Schnurr 2010, S. 347). Fehlt dabei ein klares, allgemeingültiges Verständnis von Nachhaltigkeit, kann sich Opportunität breit machen (Mast und Fiedler 2007, S. 569f., 575).

Dies gilt besonders in Zeiten des Wandels, wenn eine strategische Neuausrichtung im Sinne der Nachhaltigkeit stattfindet. Die Integration von Nachhaltigkeitsthemen in die Strategie und damit in die Identität des Unternehmens, wie sie in diesem Beitrag beleuchtet wird, steht in Verbindung mit tiefgreifenden Veränderungen, deren Management und Kommunikation (Walter 2017, S. 103, 112). Geplante strategische Wandlungsprozesse, die durch die Nachhaltigkeitsorientierung initiiert werden, stellen keine Projekte mit definiertem Start- und Endpunkt dar, wie es im Veränderungsmanagement meist der Fall ist. Sie haben den Charakter von grundlegenden Denk-, Verhaltens-, Führungs-, 
Arbeits- und Produktionsweisen (Wagner 2019, S. 121). Den Wandel zielorientiert zu steuern und zu gestalten bedeutet, die vier Handlungsfelder Strategie, Struktur, Kultur und Individuum in den Blick zu nehmen (Lauer 2019, S. 7).

In ihrer Funktion als ,motor of change“ (Carollo und Guerci 2017, S. 632, 637) kann Nachhaltigkeit das Unternehmen fundamental verändern. Für die gezielte Steuerung dieses Unternehmenswandels durch das Veränderungsmanagement (Lauer 2019, S. 3 f.; Rank und Scheinpflug 2010, S. 18f.) spielt neben den harten Faktoren auch der ,Faktor Mensch“ eine wesentliche Rolle (Merten 1999, S. 51). Er bringe ,[...] starke Implikationen für eine intensive Kommunikation" mit sich, schreiben Buchholz und Knorre (2019, S. 245). Ähnlich betont Walter (2017, S. 111), dass es angesichts des moralischen Anspruchs, der mit diesem besonderen Veränderungstyp einhergeht, für die Verankerung und Operationalisierung einer auf Nachhaltigkeit gerichteten Neuausrichtung viel Zeit und einer umfassenden kommunikativen Begleitung bedürfe. Darin klingen Mitarbeitende als Bezugsgruppe der internen Kommunikation an. Bis dato fehlen aber v.a. empirische Erkenntnisse zu den „Implikationen“ (Buchholz und Knorre 2019, S. 245), die Kommunikationsfachleute in diesem Zusammenhang wahrnehmen und wie sie insbes. die Rollen und Funktionen, die Mitarbeitende als Zielgruppe und Kommunikationspartner in diesem Veränderungstyp einnehmen können, verstehen. Als erste Forschungsfrage lässt sich formulieren: Welche Funktion und Rollen schreiben Kommunikationsfachleute den Mitarbeitenden bei der strategischen Neuausrichtung im Sinne der Nachhaltigkeit zu? Handelt es sich um dieselben Funktionen und Rollen, die in Strategieprozessen i. A. genannt werden, oder stehen Funktionen und Rollen in spezifischem Zusammenhang zur Nachhaltigkeitsthematik? Im Kontext einer Nachhaltigkeitsstrategie, die von (veränderten) Werten getragen sein muss und sich ggf. auch in einem Wandel der gemeinsamen Wertanschauungen niederschlagen kann, kommt dem Verständnis, der Akzeptanz und der Übernahme der Werte ins Verhalten der Mitarbeitenden eine besondere Bedeutung zu. Vor diesem Hintergrund ist die Frage nach Funktion und Rollen in nachhaltigkeitsorientierten Veränderungsprozessen und die damit verbundenen Ziele und Aufgaben der internen Nachhaltigkeitskommunikation ein erster wesentlicher Ausgangspunkt.

\subsection{Ziele und Aufgaben interner Kommunikation im Veränderungsprozess}

Veränderungsmanagement bedeutet, potenzielle Verhaltensweisen der Mitarbeitenden zu antizipieren und Akzeptanz, Einstellungs- und Verhaltensänderungen zu fördern (Merten 1999, S. 51). Rank und Scheinpflug (2010, S. 18f.) definieren Change- bzw. Veränderungsmanagement als ,,[...] die Planung, Implementierung, Kontrolle und Stabilisierung der
Veränderungen in Strategien, Prozessen, Organisation und Kultur mit dem Ziel, die Effektivität und Effizienz des Veränderungsprozesses zu maximieren und die größtmögliche Akzeptanz der betroffenen Führungskräfte und Mitarbeiter zu erreichen“. Merten (1999, S. 51) konkretisiert dieses Verständnis, indem er schreibt, dass Veränderungsmanagement auf ,die planmäßige, mittel- bis langfristige wirksame Veränderung der Verhaltensmuster und Einstellungen von Organisationsmitgliedern" abziele. In der Literatur werden in diesem Zusammenhang sowohl die Veränderungsbereitschaft, die auf inneren Einstellungen oder einem Nutzenkalkül beruht (Einstellungsakzeptanz), als auch die Neigung, aktiv am Wandel teilzunehmen (Verhaltensakzeptanz), genannt (Krüger 2014, S. 19). Bei der Implementierung von Nachhaltigkeit gilt es, diese Einstellungs- und Verhaltensakzeptanz in Bezug auf Nachhaltigkeitsorientierung und Verantwortungsübernahme auszuformen (Karst et al. 2000, S. 47).

Wandelvorhaben brauchen demnach Kommunikation. Der Veränderungskommunikation i. A. werden hierbei drei Kernaufgaben zugeschrieben (Deutinger 2017, S. 10): die Informationsvermittlung, d.h. der gezielte Informationsaustausch und das Schaffen von Transparenz über die Absichten, die Notwendigkeit, die Auswirkungen und den Status Quo der Veränderung (Lauer 2010, S. 108); Partizipation und Involvement im Sinne der aktiven Integration, Mobilisierung und Motivation der Betroffenen, sich eigenständig in den Veränderungsprozess einzubringen (ZowisloGrünewald und Schwab 2003, S. 7) sowie zur Aufnahme von Emotionen und Bearbeitung von Widerständen (Kaune und Wagner 2016, S. 39); und schließlich die Beratung und Befähigung der Führungskräfte, die erste Anlaufstelle für die ihnen zugeordneten Mitarbeitenden und lebendiges Vorbild für die Realisierung der Veränderung sein sollten (Kleinfeld und Schnurr 2010, S. 358). Während die ersten beiden klar als Zielsetzungen interner Veränderungskommunikation bezeichnet werden können, klingt in der dritten Aufgabe bereits ein Instrument - die Führungskräftekommunikation und deren besondere Bedeutung in Veränderungssituationen - mit an. Betrachtet man die interne Kommunikation i.e.S., so werden in der Literatur als weitere Aufgaben auch die Steigerung der Motivation der Mitarbeitenden, den Wandel mitzutragen oder gar aktiv mitzugestalten, die Stärkung des Commitments innerhalb der Organisation (Engert und Baumgartner 2016, S. 822, 830f.) und die Orientierung (Huck-Sandhu 2013; Buchholz und Knorre 2019, S. 248) genannt.

Ein Veränderungsprozess hin zu (mehr) unternehmerischer Nachhaltigkeit und Verantwortung weist - wie oben dargestellt - besondere Merkmale auf, die ihn von klassischen Strategieprozessen in wesentlichen Merkmalen unterscheiden. Er ist gekennzeichnet durch eine besonders hohe Themenkomplexität, ist oftmals normativ belegt und 
beinhaltet per se einen moralischen Anspruch. Diese Rahmenbedingungen, so lässt sich als These formulieren, finden Eingang in die spezifische Aufgabendefinition der internen Kommunikation und sind so auch eng mit den Zielen, die die strategische Kommunikation im Veränderungsprozess verfolgt, verbunden. Als zweite Forschungsfrage kann formuliert werden: Welche spezifischen Ziele und Aufgaben verfolgt interne Veränderungskommunikation bei der Etablierung neuer Nachhaltigkeitsstrategien? In diesem $\mathrm{Zu}-$ sammenhang soll beleuchtet werden, ob sich jene Ziele und Aufgaben, die in der Literatur für Veränderungs- und Strategieprozesse allgemein genannt werden, in identischer Form, Ausprägung und Gewichtung auch im Kontext neuer Nachhaltigkeitsstrategien zeigen. Zudem lässt sich die Frage stellen, ob ggf. spezifische Ziele und Aufgaben oder Ausprägungen davon aus dem Kontext der internen Nachhaltigkeitskommunikation hinzutreten, die in thematisch andersgelagerten Veränderungsprozessen bislang nicht beobachtet worden sind. Je nach Zusammenspiel können sich hieraus neuartige Anforderungen an den Einsatz und die Gestaltung der internen Nachhaltigkeitskommunikation im Veränderungsprozess ergeben.

\subsection{Kommunikative Implementation strategischer Neuausrichtung}

Im Rahmen der Implementation der internen Nachhaltigkeitskommunikation während des Veränderungsprozesses gilt es, Entscheidungen über die Art und Weise zu treffen, wie Emotionen und Kognitionen - also Herz und Verstand - angesprochen werden können. Auch die geeigneten Maßnahmen und Instrumente, mit denen die neuen Strategiebestandteile in den bestehenden kognitiven, affektiven und konativen Mustern der Mitarbeitenden verankert werden können, müssen ausgewählt werden (Raps 2017, S. 27): „capturing the hearts and minds of all employees“, schreiben Dunphy et al. (2007, S. 93) in diesem Zusammenhang. Grundlage sind - analog zu den Kernaufgaben - die Vermittlung einer breiten Wissensbasis, die Stärkung des Commitments (Dolphin 2005, S. 174) und die Angleichung der affektiven und konativen Muster an die strategischen Voraussetzungen (Kipping und Nierstenhöfer 2009, S. 148). Dazu bedarf es der Offenheit der Führungskräfte und Mitarbeitenden für Neues sowie ihrer Bereitschaft zur Veränderung (Reiners 2015, S. 97), aber auch einer glaubwürdigen Zukunftsperspektive.

Im Rahmen der allgemeinen Veränderungskommunikation werden die Instrumente und Maßnahmen interner Kommunikation möglichst breit genutzt und ganzheitlich eingesetzt. Als wirksamstes Instrument gilt dabei die persönliche Kommunikation, weil sie als reicher Kommunikationskanal (Daft und Lengel 1986) komplexe Inhalte verständlich, umfassend und vertrauensfördernd im direkten Dia- log vermitteln kann (Buchholz und Knorre 2019, S. 246; Mast 2008, S. 21). Im Mehrdimensionen-Themenfeld der Nachhaltigkeit erscheint dies besonders wichtig. Im Rahmen der persönlichen Veränderungskommunikation kommen u.a. Townhall-Meetings, Schulungen, Besprechungen oder Mitarbeitergespräche in Frage. Aber auch die begleitende digitale und gedruckte Kommunikation ist wichtig, um breit zu informieren: als aktuelle Information z. B. im Intranet oder in Form der Hintergrundberichterstattung etwa in der Mitarbeiterzeitschrift (Zowislo-Grünewald und Schwab 2003, S. 68f.).

In der Literatur wird darauf hingewiesen, dass insbesondere Storytelling für die Nachhaltigkeits- und Veränderungskommunikation gut geeignet sei (Wagner 2019, S. 89). Lebendige Geschichten, Metaphern und ein roter Faden unterstützen die Erläuterung komplexer Veränderungen und die Strategieimplementierung (Deutinger 2016, S. 100). Im Rahmen des Storytellings als narrativem Modus (Lünenborg 2005, S. 126, 159) werden die auf Nachhaltigkeit ausgerichteten strategischen Werte, die Unternehmenskultur, die Vision, die Mission, das Leitbild, erwünschte Einstellungs- und Verhaltensmuster sowie motivationale Faktoren in Geschichten übersetzt (Buchholz et al. 2019, S. 37). Mithilfe von Analogien aus der Lebens- und Alltagswelt der Mitarbeitenden werden die wesentlichen Bestandteile des Wandels in die Vorstellungs- und Verstehenswelt der Mitarbeitenden überführt (Mast 2016, S. 476) und mit Assoziationen und Emotionen belegt (Kipping und Nierstenhöfer 2009, S. 154). Somit spricht narrative Kommunikation Emotion und Kognition gleichermaßen an.

Im Rahmen der internen Veränderungskommunikation gilt es grundsätzlich, den langfristigen Nutzen und die Sinnhaftigkeit der angestrebten Verhaltensänderung zu vermitteln (Berner 2012, S. 95) - eine Aufgabe, die auch als Sensemaking bezeichnet wird (Weick 1985, 1995) und im Kontext von Nachhaltigkeit besonders relevant erscheint (Cramer et al. 2006, S. 382). Studien zeigen, dass sich Menschen in Bezug auf ihre Ziele, Werte, Interessen sowie Menschenbilder, subjektiv betrachtet, immer sinnstiftend verhalten. Sie verändern ihr Verhalten nur, wenn das neue Verhalten eine höhere Wahrscheinlichkeit der Zielerreichung aufweist (Berner 2012, S. 120).

Weitet man die Perspektive im Kontext der Implementation, so stellt lässt sich eine dritte Forschungsfrage formulieren: Wie wird die interne Nachhaltigkeitskommunikation bei der strategischen Neuausrichtung im Sinne der Nachhaltigkeit eingesetzt und ausgestaltet? Ausgehend von Erkenntnissen zur Veränderungskommunikation i. A. stellt sich die Frage, ob der möglichst breite, ganzheitliche Einsatz von persönlich, digitalen und geruckten Kommunikationsinstrumenten und -medien auch im Rahmen interner Nachhaltigkeitskommunikation zur Implementation einer neuen Nachhaltigkeitsstrategie zum Tragen kommt. Neben 
der Art und Ausprägung von narrativer Kommunikation soll besonderes Augenmerk auch auf der Frage nach der Sinnhaftigkeit sowie möglichen zusätzlichen, spezifisch im Bereich Nachhaltigkeit relevanten Maßnahmen, Instrumenten und Vermittlungstechniken liegen.

\subsection{Rahmenfaktoren und Herausforderungen der Strategieimplementierung}

Organisationsstruktur, Werte sowie die Unternehmens- und Führungskultur bilden Rahmenfaktoren für die Art und Umsetzung der Veränderungskommunikation und ihrer Wirksamkeitspotenziale (Engert und Baumgartner 2016, S. 822, 830 f.; Mast 2016, S. 476; Kleinfeld und Kettler 2017, S. 89f.; Wieland 2004, S. 14; Kleinfeld und Schnurr 2010, S. 327). Sie können auch als Kontextfaktoren bezeichnet werden, die in strategischen Veränderungsprozessen einen festen und dauerhaften, aber zugleich auch veränderbaren Rahmen bilden. Besonders für die Etablierung einer Nachhaltigkeitsstrategie erscheinen sie wesentlich, weil nachhaltige Unternehmensführung im normativen Kern einer Organisation ansetzt.

Durch das Ausrollen neuer Werte im Unternehmen wird sichergestellt, dass die neue Strategie in allen Unternehmensbereichen und auf allen Ebenen präsent ist (Maon et al. 2009, S. 78). Um die für Nachhaltigkeit relevanten Denk- und Handlungslogiken zu etablieren, bedarf es ihrer Vermittlung und fortwährenden Aktualisierung auch mittels einer unternehmensweiten, kontinuierlichen Kommunikation dieser Werte (Buchholz et al. 2019, S. 1, 3, 15). Wo immer diese Werte für Mitarbeitende handlungs- und entscheidungsrelevant sind, kommt es in besonderer Art und Weise darauf an, diese verständlich und für den individuellen Arbeitsbereich greifbar zu machen. Dazu werden sie auf die verschiedenen Managementebenen heruntergebrochen (Wieland 2004, S. 26f.). Die Werte manifestieren sich zudem in der Unternehmenskultur (ebd., S. 14; Kleinfeld und Kettler 2017, S. 88), die Treiber und Anker für die Veränderung des Mitarbeiterverhaltens zugleich ist: Durch ihren Wandel verändert sich einerseits der Unternehmensalltag, andererseits wird das Neue gerahmt, konsolidiert und routinisiert (Berner 2012, S. 66). Die Unternehmenskultur stiftet gemäß ihrer Primärfunktion Ordnung, Orientierung, Identität und Sinn (Sackmann 2019, S. 5f.). Die interne Nachhaltigkeitskommunikation unterstützt die enge Verflechtung von Strategie und Unternehmenswerten, indem sie Sensemaking- und Sensegiving-Prozesse bei den Mitarbeitenden begünstigt, die Veränderung der Unternehmenskultur im Sinne des Wandels vorantreibt und ein kommunikatives Wertemanagement betreibt. Das adäquate Austarieren dieser Faktoren ist grundlegend, um Nachhaltigkeit zum Maßstab des Handelns der Mitarbeitenden werden zu lassen und den Wandel zu implementieren (Kleinfeld und Schnurr 2010, S. 327).

Neben den kulturellen Faktoren bedarf es auch institutioneller Rahmenbedingungen, damit die Einführung und Durchsetzung einer Nachhaltigkeitsstrategie gelingen kann. Für die Strategieimplementierung kommt hier v.a. den Führungskräften eine zentrale Rolle zu, wie im Kontext der Kernaufgaben interner Veränderungskommunikation bereits angeklungen ist. Sie sind Kommunikator, Dialogpartner, Multiplikator und Vorbild (Dunphy et al. 2007, S. 217; Prexl 2010, S. 350; Engert und Baumgartner 2016, S. 829). Die Führungskräftekommunikation selbst nimmt im Veränderungsprozess im Wesentlichen zwei Funktionen ein: Sie flankiert und stützt andere Kommunikationskanäle und -instrumente, stellt aber auch einen eigenständigen Kanal für die Verteilung von Managementinformationen dar (Mast und Huck 2008, S. 156). Im Sinne der transformationalen Führung ist es ihre Aufgabe, eine überzeugende Vision und Mission zu entwickeln, gemeinsame Ziele zu setzen, Vertrauen zu vermitteln, eine kollektive Identität zu kreieren, Emotionen zu wecken und zum Engagement für das allgemeine Wohl zu inspirieren und $\mathrm{zu}$ motivieren (Allen 2016, S. 155). Führungskräfte sind zudem Initiatoren, Promotoren und unternehmensinterne Treiber, aber eben auch selbst Betroffene nachhaltigkeitsorientierter Veränderungsvorhaben (Gelbmann und Baumgartner 2012, S. 292; Deutinger 2016, S. 100). Sie können als „Seismographen“ von Wahrnehmungslagen und Befindlichkeiten der Mitarbeitenden in den Veränderungsprozess eingebunden werden, aber agieren im Idealfall auch als Change Agents bzw. Promotoren, die Weichensteller im Prozess sind (Dunphy et al. 2007, S. 19).

Bezüglich Rahmenfaktoren interner Veränderungskommunikation lässt sich somit eine vierte Forschungsfrage ableiten: Inwiefern tragen kulturelle und institutionelle Parameter zur kognitiven und affektiven Verankerung sowie zur Operationalisierung der Strategie bei?

Aus den Rahmenfaktoren heraus kann eine fünfte, übergeordnete Forschungsfrage formuliert werden: Worin bestehen die zentralen Herausforderungen bei der Strategieimplementierung durch Kommunikation aus Sicht der Kommunikatoren? Allgemeine und spezifische Herausforderungen, die in der Unternehmenspraxis bestehen, bilden - ähnlich wie die o.g. internen Rahmenfaktoren einen Orientierungspunkt, wenn es darum geht, wie interne Veränderungskommunikation bei der Etablierung von Nachhaltigkeitsstrategien gestaltet werden kann. Sie können sowohl kommunikationsspezifische Herausforderungen als auch Herausforderungen anderer Natur, die auf kommunikative Aspekte der Veränderungskommunikation im Nachhaltigkeitskontext einwirken, beinhalten. 


\section{Empirische Untersuchung}

Die Literaturanalyse bildet den Ausgangspunkt für die empirische Auseinandersetzung mit der Frage, welche Grundlagen, Rahmenbedingungen und Erfolgsfaktoren für die interne Veränderungskommunikation in strategischen Veränderungsprozessen zur Etablierung einer neuen Nachhaltigkeitsstrategie identifiziert werden können.

FF1 Welche Funktion und Rollen schreiben Kommunikationsfachleute den Mitarbeitenden bei der strategischen Neuausrichtung im Sinne der Nachhaltigkeit zu?

FF2 Welche spezifischen Ziele und Aufgaben verfolgt interne Veränderungskommunikation bei der Etablierung neuer Nachhaltigkeitsstrategien?

FF3 Wie wird die interne Nachhaltigkeitskommunikation bei der strategischen Neuausrichtung im Sinne der Nachhaltigkeit eingesetzt und ausgestaltet?

FF4 Inwiefern tragen kulturelle und institutionelle Parameter zur kognitiven und affektiven Verankerung sowie zur Operationalisierung der Strategie bei?

FF5 Worin bestehen die zentralen Herausforderungen bei der Strategieimplementierung durch Kommunikation aus Sicht der Kommunikatoren?

Zur Untersuchung dieser fünf Forschungsfragen wird ein qualitativer Ansatz gewählt. Ziel ist es, die Gegebenheiten in der Unternehmenspraxis in ihrer ganzen Breite erfassen und sich dem bislang wenig untersuchten Arbeitsfeld explorativ annähern zu können (Steffen und Doppler 2019, S. 9; Brosius et al. 2016, S. 4).

\subsection{Anlage und Umsetzung der Studie}

Aufgrund des explorativen Charakters des Studiendesigns wird zur Auswertung der leitfadengestützten Experteninterviews auf die Methode der induktiven qualitativen Inhaltsanalyse zurückgegriffen (Mayring 2010, S. 83). Mithilfe von Leitfadeninterviews mit Verantwortlichen für interne Nachhaltigkeitskommunikation - also denjenigen Kommunikationsmanager*innen, die für die kommunikative Begleitung des Veränderungsprozesses verantwortlich sind sollen individuelle, subjektive und detailreiche Begründungen in Form von persönlichen Einschätzungen, Meinungen und Erklärungen erhoben werden und in Relation zu den theoretischen Erkenntnissen des vorangegangenen Kapitels gesetzt werden (ebd., S. 4, 107; Kaiser 2014, S. 5f.).

Die Leitfadeninterviews waren als strukturierte und gesteuerte Gespräche konzipiert, jedoch mit Raum für individual-spezifische Nach- und Detaillierungsfragen zur Vertie- fung bestimmter Aspekte angelegt (Mey und Mruck 2011, S. 271). So konnten sowohl die Vergleichbarkeit der Themen als auch die individuelle Wortwahl durch die Befragten gewährleistet werden (ebd.; Steffen und Doppler 2019, S. 30). Die ersten beiden Interviews waren als Pretest angelegt. Der Leitfaden erwies sich als verständlich, eindeutig und reliabel, so dass keine Anpassungen notwendig wurden und diese Interviews in die Stichprobe mit einfließen konnten. Die leitfadengestützten Experteninterviews wurden entlang des Gesprächsleitfadens (siehe Anhang, Interviewleitfaden) geführt, digital aufgezeichnet und transkribiert. Aufgrund des explorativen Charakters des Studiendesigns wurde für die Auswertung auf die Methode der induktiven qualitativen Inhaltsanalyse zurückgegriffen (Mayring 2010, S. 83). Bei der Lektüre werden aus den Inhalten des Textmaterials systematisch intersubjektive Kategorien gebildet, ohne dabei Bezug zu vorab thematisierten theoretischen Konstrukten zu nehmen (ebd.; Brosius et al. 2016, S. 17). Vorteil der induktiven Kategorienbildung gegenüber dem theoriegestützten, deduktiven Vorgehen ist die Darstellung der Analyseergebnisse ohne Verzerrung durch theoretische Vorannahmen (Mayring 2010, S. 84). Anhand der gebildeten Kategorien - u. a., die Bedeutung der internen Kommunikation von Nachhaltigkeit, die Ziele der internen Nachhaltigkeitskommunikation, die Rolle der Mitarbeitenden bei der strategischen Neuausrichtung sowie die inhaltliche Aufbereitung von Nachhaltigkeitsthemen - wurden im Anschluss Rohdaten extrahiert, kategorisiert, aufbereitet, ausgewertet und mit den theoretischen Erkenntnissen verknüpft (Gläser und Laudel 2009, S. 199). Dies erfolgte mithilfe einer Tabelle, in der Interviewpassagen aus den Gesprächen (Spalten) den vorab bestimmten Kategorien (Zeilen) zugeordnet werden. Die Zuordnung der Interviewpassagen erfolgte mittels eines Kodierleitfadens (siehe Anhang, Tab. 2). Darauf aufbauend wurde eine qualitative Analyse des Interviewmaterials durchgeführt. Innerhalb der zuvor gebildeten Kategorien werden Gemeinsamkeiten, Unterschiede sowie Widersprüche herausgearbeitet und zusammengefasst. Anschließend wurden die Inhalte aus den verschiedenen Gesprächen je Kategorie verglichen und ausgewertet. Dabei stand die explorierende Interpretation der Inhalte und weniger das Erfassen von Häufigkeiten im Fokus.

Grundlage der Studie war eine kleine, anhand fester Kriterien ausgewählte, heterogene Stichprobe (Steffen und Doppler 2019, S. 17 f.). Die Kriterien wurden auf Grundlage einer Online-Recherche auf Unternehmens-Websites und dem CSR-Benchmark $2019^{1}$ festgelegt. Die ausgewählten

\footnotetext{
${ }^{1}$ Basierend auf der ,Studie zur digitalen Nachhaltigkeitskommunikation großer Unternehmen in Deutschland“, https://www.csrbenchmark.de/corporate-social-responsibility-benchmark-2019/ benchmark.
} 
Tab. 1 Befragte Kommunikationsexpert*innen

\begin{tabular}{llll}
\hline Unternehmen & Nr. & Position/Funktion Interviewpartner & Branche \\
\hline Bayer AG & $\# 1$ & Communications Sustainability \& CSR/Spokesman & Life-Science/Pharma \\
Deutsche Bahn AG & $\# 2$ & Interne Kommunikation & Verkehr und Logistik \\
Flughafen München GmbH & $\# 3$ & Unternehmenskommunikation, Konzernbereich - Nachhaltigkeit & Verkehr und Logistik \\
Ford Werke GmbH Deutschland & $\# 4$ & Leiterin Unternehmenskommunikation & Automobil \\
Kärcher SE \& Co. KG & $\# 5$ & Interne Kommunikation Corporate Communications, Products \& Chan- & Reinigungstechnik \\
& & nels, Corporate Marketing \& Brand Management & Nahrung \\
McDonald's DE GmbH & $\# 6$ & Manager External Communication/Corporate Affairs & Nahrung \\
Nestlé AG Deutschland & $\# 7$ & Sustainability Manager & Nahrung \\
Unternehmen A (anonymisiert) & $\# 8$ & PR-Manager Wirtschaft \& Nachhaltigkeit & Logistik \\
Schnellecke Group AG \& Co KG & $\# 9$ & Head of Group Communications, Employer Branding \& Marketing & Hightech \\
Unternehmen B (anonymisiert) & $\# 10$ & Senior Manager Sustainability &
\end{tabular}

Unternehmen hatten bestimmte Voraussetzungen zu erfüllen: (1) Ein Unternehmen sollte in Deutschland tätig sein, so dass vergleichbare Aussagen zur Praxis der internen Nachhaltigkeitskommunikation unter den gegebenen nationalen Rahmenbedingungen möglich sind. (2) Es soll sich um ein Großunternehmen bzw. großes mittelständisches Unternehmen handeln, um sicherzustellen, dass einschlägige Kommunikationsfunktionen vorhanden sind. Die Branche hingegen wurde als nicht relevantes Auswahlkriterium eingestuft, da die Etablierung von Nachhaltigkeitsstrategien aktuell in vielen Branchen einen hohen Stellenwert einnimmt; sie wurde im Rahmen der Auswertung aber mitgeführt, um Einflüsse der Unternehmensauswahl mit kontrollieren zu können. (3) Die Auseinandersetzung des Unternehmens mit dem Thema Nachhaltigkeit wird auf der UnternehmensWebsite (z.B. Website allgemein, Newsroom oder Media Center) deutlich hervorgehoben. (4) Das Unternehmen befindet sich gegenwärtig oder befand sich in der nahen Vergangenheit in einem Veränderungsprozess der strategischen Neuausrichtung in Richtung unternehmerische Nachhaltigkeit und Verantwortung, der anhand der externen Kommunikation des Unternehmens selbst, der Medien- und Branchenberichterstattung oder sonstiger verlässlicher OnlineQuellen, etwa zu Auszeichnungen und Preisen, über eine Online-Recherche von d. Verf. zu identifizieren waren.

Im Rahmen der Recherche wurden als Ergebnis einer solch bewussten Auswahl 96 in Deutschland tätige Unternehmen identifiziert, die die o.g. Kriterien eindeutig erfüllten bzw. für die auf Grundlage der Recherche zu erwarten war, dass diese Kriterien mit großer Wahrscheinlichkeit ${ }^{2}$ zutreffend sind. In diesen Unternehmen wurden jene Kommunikationsexpert*innen, die für Interne Kommunikation,

\footnotetext{
${ }^{2}$ Ein Beispiel: Die externe Recherche ergab eine laufende strategische Neuausrichtung hin zu unternehmerischer Nachhaltigkeit und Verantwortung, jedoch war nicht eindeutig erkennbar, wie weit der Prozess bereits vorangeschritten und in welcher Phase die Umsetzung und interne Kommunikation sich befanden.
}

Veränderungskommunikation und/oder das Thema Nachhaltigkeit verantwortlich waren, über eine Internetrecherche ermittelt, schriftlich kontaktiert und für die Teilnahme an der Studie angefragt. Wo die bzw. der jeweils zuständige Expert*in im Rahmen der Web-Recherche nicht identifiziert werden konnte, erfolgte der Erstkontakt über die Pressestelle oder anderweitig ausgewiesene zuständige Ansprechpersonen mit Bitte um Weitergabe der Anfrage bzw. Kontaktvermittlung. Von den 96 kontaktierten Unternehmen erklärten sich zehn Kommunikationsexpert*innen zu einer Teilnahme bereit. Nach näherer Prüfung, dass die o.g. inhaltlichen Auswahlbedingungen für die jeweilige Organisation und Kommunikationsarbeit zutrafen, wurden die Experteninterviews im Januar 2020 telefonisch geführt (Tab. 1). Aufgrund der ergänzenden Informationen, die durch die VorabRecherche und im Rahmen der Interviews zum jeweiligen Veränderungsprozess gewonnen wurden, konnten die Aussagen in den Kontext des Strategieprozesses eingeordnet werden. Dadurch ergab sich ein fallstudienartiger Zugang zur Erhebung und Auswertung. Acht der zehn befragten Expert*innen gaben ihr Einverständnis für die Publikation ihrer Funktion und ihres Unternehmens, zwei Befragte baten um Anonymisierung.

\subsection{Ergebnisse}

Die Analyse und Interpretation der Ergebnisse aus Leitfadengesprächen werden im Folgenden entlang der vier Leitfragen, die sich aus der Literaturdurchsicht ableiten, strukturiert und interpretiert:

\subsubsection{FF1: Welche Funktion und Rollen schreiben Kommunikationsfachleute den Mitarbeitenden bei der strategischen Neuausrichtung im Sinne der Nachhaltigkeit zu?}

Bei den befragten Expert*innen für interne Nachhaltigkeitskommunikation herrscht Konsens darüber, dass die Mitar- 
beitenden von essenzieller Bedeutung für den Erfolg einer strategischen Neuausrichtung im Sinne der Nachhaltigkeit sind. Sie bestätigen hiermit die theoriebasierte Annahme, dass Mitarbeitende im Wandel eine wesentliche Rolle spielen. Entsprechend werde die interne Kommunikation im Strategieprozess von Beginn an mitgedacht und eingebunden. „Im Prinzip dreht sich alles um die Mitarbeitenden. Die gesamte Strategie wurde um sie herum entwickelt“", hält ein Kommunikator der Bayer AG (\#1) fest.

Sichtbar wird diese besondere Bedeutung der Mitarbeitenden einerseits an der Tatsache, dass eine Einbindung von Mitarbeitenden in den Prozess der Strategieformulierung zu einem frühen Zeitpunkt und über alle Hierarchieebenen hinweg stattfindet, wie Engert und Baumgartner (2016, S. 830) betonen. Andererseits kommt sie in den differenzierten Rollen bzw. Funktionen, die den Mitarbeitenden für die strategische Neuausrichtung von Nachhaltigkeitsstrategien zugesprochen wird, zum Ausdruck:

Mitarbeitende können als Implementoren der Strategie angesehen werden, da sie die Strategie im täglichen Arbeiten mit Leben füllen und umsetzen. Tragen sie in ihrem Verhalten die Nachhaltigkeitsorientierung nicht mit, ,wird das Ganze ungleich schwieriger", wie eine Mitarbeiterin der internen Kommunikation bei Kärcher (\#5) betont. Bereits anhand der beschriebenen Bedeutung des Verhaltens der Mitarbeitenden zeichnet sich der von Wagner (2019, S. 34) herausgearbeitete Stellenwert der internen Kommunikation in diesen spezifischen Wandlungsprozessen ab: Ohne sie ist die Umsetzung kaum möglich.

Mitarbeitende sind zudem interne Multiplikatoren, da sie als Kommunikatoren sowohl auf der formellen als auch auf der informellen Kommunikationsebene Informationen weitergeben sowie Meinungsbildungs- und Adaptionsprozesse auslösen. In der Unternehmenskommunikation zum Thema Nachhaltigkeit des Flughafens München (\#3) gilt: „Wenn man möglichst viele Leute für das Thema begeistern kann, hat man schon halb gewonnen".

Mitarbeitenden wird zudem die Rolle als Botschafter des Wandels nach außen zugeschrieben. Sie sollten z.B. im Kundenkontakt korrekt und umfassend erklären können, wie das nachhaltige und verantwortungsvolle Wirtschaften des Unternehmens angelegt ist, so die Befragten. In dieser externen Rolle beeinflussen sie die Wahrnehmung, das Image und das Nachhaltigkeitsengagement des Unternehmens, was sich u. a. auch in einer höheren Glaubwürdigkeit niederschlagen kann.

Somit bestätigen die befragten Kommunikator*innen, dass Mitarbeitende im Kontext des strategischen Wandels nicht nur umsetzende Instanz sind, sondern als Change Agents, Botschafter und Multiplikatoren fungieren können, die wesentliche Akteure im Wandel sind (vgl. etwa Prexl 2010, S. 374; Carollo und Guerci 2017, S. 637).

\subsubsection{FF2: Welche spezifischen Ziele und Aufgaben verfolgt interne Veränderungskommunikation bei der Etablierung neuer Nachhaltigkeitsstrategien?}

In den Gesprächen zeigt sich ein weitgehend homogenes Bild: Als zentrale Ziele gelten die Steigerung des Wissensstandes durch Information, die Schaffung von Akzeptanz, Motivation und Handlungsbereitschaft und das Einbinden bzw. Involvieren der Mitarbeitenden. Innerhalb dieser Oberziele werden die Sensibilisierung der Mitarbeitenden für die Relevanz der Nachhaltigkeitsthematik insgesamt, aber auch für die Bedeutung, die dem individuellen Beitrag eines jeden Mitarbeitenden hier zukommt, genannt. Zudem wird betont, dass die Kommunikation der (Hinter-)Gründe, Rahmenbedingungen, des Zwecks und Sinns sowie der Auswirkungen unternehmerischer Nachhaltigkeit in einem solchen Prozess essenziell sei.

Zwei Befragte (\#7, \#9) ergänzen als weiteres Ziel die Befähigung der Mitarbeitenden. Es gelte, Mitarbeitende nicht nur ausreichend zu informieren, sondern sie gerade in Zeiten der strategischen Veränderung gezielt in die Veränderung mit einzubinden. Das „Abholen der Mitarbeitenden“ (\#8) wird als wesentlich erachtet.. Die Interviews machen deutlich, dass interne Kommunikation in den befragten Unternehmen die emotionale Disposition der Mitarbeitenden aufzunehmen versucht, sie informiert und aktiviert und zur aktiven Teilhabe aufruft. Kommunikative Hebel sind hier der Stolz auf die künftige nachhaltige Ausrichtung des Unternehmens, der Wunsch, selbst sinnstiftend zu arbeiten und selbst mitgestalten zu können.

Entsprechend gehen die Aufgaben der internen Kommunikation zu Nachhaltigkeit weit über das reine Informieren hinaus. Damit werden die von Deutinger $(2017$, S. 10) identifizierten drei kommunikativen Kernaufgaben im Wandel in der Praxis ganzheitlich bedient. Die bei Nestlé Deutschland (\#7) beschäftigte Kommunikationsverantwortliche betont: „Man muss Mitarbeiter abholen, motivieren, sie aber auch aktiv involvieren". Dabei bilden die Antworten der Befragten eine breite Spanne der Beteiligung ab, von der Möglichkeit zum aktiven Einbringen von Ideen (\#2) und Verbesserungsvorschlägen bis hin zur proaktiven Mitarbeit in der Strategieentwicklung (\#1).

Zudem wird in den Leitfadengesprächen deutlich, dass Mitarbeitende für die Relevanz der Nachhaltigkeitsthematik und für die Bedeutung eines eigenen Beitrags sensibilisiert werden sollten (\#2,\#4, \#5, \#6, \#9). Die Kommunikation der Inhalte, der Gründe, des Zweckes und Sinns sowie der Auswirkungen und Rahmenbedingungen der Veränderung gilt als wesentlich für die Schaffung von Verständnis. Jedoch muss das Interesse der Mitarbeitenden, sich mit der Nachhaltigkeitsthematik auseinanderzusetzen, überhaupt erst geweckt werden. Dabei gilt es auch, etwaige „Berührungsängste“ zu überwinden, wie eine Mitarbeiterin der Perso- 
nalkommunikation der Deutschen Bahn (\#2), unterstreicht. Dies deutet darauf hin, dass die Sensibilisierungsphase, wie sie Maon et al. (2009, S. 76) in ihrem Change ManagementFramework ergänzen, im Kontext von Nachhaltigkeitsthemen besondere Bedeutung haben könnte.

Somit bestätigen sich die allgemeinen Grundlagen der Veränderungskommunikation: Über die verschiedenen Ziele und Aufgaben interner Nachhaltigkeitskommunikation vollziehen sich Wissens-, Einstellungs- und Verhaltensänderungen im Veränderungsprozess in unterschiedlichen Ausmaßen, zu unterschiedlichen Zeitpunkten und in Abhängigkeit von der charakterlichen Disposition des Individuums. Mitarbeitende werden bezüglich unterschiedlicher Motivationsfaktoren, die insbes. die emotionale Disposition tangieren, aktiviert und zum Handeln aufgerufen.

In den Interviews wurde auch die Frage der Sinnhaftigkeit (Berner 2012, S. 95) angesprochen, die in der Literatur zur internen Kommunikation im Kontext von Sensemaking sowie -giving (Quirke 2008, S. 29; Schultz und Wehmeier 2011, S. 380) diskutiert wird. Interne Kommunikation, so betonen einige der befragten Kommunikationsexpert*innen (\#1, \#7, \#9, \#8, \#10), habe im Rahmen von Nachhaltigkeit immer auch den Auftrag, so zu informieren und zu kommunizieren, dass die Mitarbeitenden Sinnhaftigkeit in der strategischen Neuausrichtung erkennen können und der Wunsch gestärkt wird, über die eigene Tätigkeit einen sinnstiftenden Beitrag zu leisten. Damit knüpfen sie unmittelbar an einen Grundsatz der Entwicklung einer Nachhaltigkeitsstrategie an (Hanke und Stark 2009, S. 510): „For CSR strategizing, sensemaking and sensegiving stimulate the creation of new ways $[. .$.$] “. Durch Sensemaking und Sensegiving$ wird der Weg in eine auf anderen Werten und Grundsätzen basierende Unternehmenszukunft aufgezeigt.

\subsubsection{FF3: Wie wird die interne}

Nachhaltigkeitskommunikation bei der strategischen Neuausrichtung im Sinne der Nachhaltigkeit eingesetzt und ausgestaltet?

Die Frage nach der Wahrnehmung der strategischen Verankerung von Nachhaltigkeit als Veränderungsvorhaben wird mehrheitlich, wenngleich teilweise zögerlich, bejaht. In drei der untersuchten Unternehmen (\#1, \#8, \#10) wird die Transformation in Richtung Nachhaltigkeit lediglich als Prozess, der mit übergeordneten, eher ökonomisch bedingten Transformationen einhergeht, angesehen. Obwohl die nachhaltigkeitsorientierte, strategische Neuausrichtung in der Praxis nicht als klassischer Veränderungsprozess verstanden wird, wird in ihrem Verlauf Veränderungskommunikation mit dem Ziel der Implementierung des Neuen betrieben. Es lässt sich schlussfolgern, dass die Verbindung zwischen der strategischen Neuausrichtung auf Nachhaltigkeit und Ver- änderungskommunikation in der Praxis eher operativ als strategisch angelegt ist.

Die von den Befragten genannten Kommunikationsanlässe gliedern sich in zwei Gruppen: die (1) proaktive (Regel-)Kommunikation, welche darauf abzielt durch die kontinuierliche Kommunikation begründeter Themen eine ganzheitliche Wissensbasis aufzubauen, sowie die (2) reaktive Kommunikation zu internen wie externen aktuellen Entwicklungen und besonderen Ereignissen (\#5). Darunter fallen externe Erwartungen und Forderungen, der öffentliche Diskurs zur Nachhaltigkeitsthematik, Ereignisse wie z.B. der Weltumwelttag oder die Fridays-for-future-Bewegung (\#2). Welche Nachhaltigkeitsaspekte aus der großen Vielfalt an potenziellen Gegenständen der internen Kommunikation schließlich zu Themen werden, wird mithilfe einer Priorisierung entschieden. Hierzu werden in den befragten Unternehmen teilweise klassische journalistische Nachrichtenwerte (Galtung und Ruge 1965; Schulz 1990) wie Relevanz, Nähe oder Dauer herangezogen, teils wird die Priorisierung anhand einer Wesentlichkeitsanalyse vorgenommen (\#10).

Hinsichtlich des Medieneinsatzes zeichnet sich die Tendenz ab, dass vorrangig digital und über eine direkte, persönliche Ansprache der Mitarbeitenden und weniger über traditionelle Formate kommuniziert wird. Als Argument für den Einsatz digitaler Medien wird genannt, dass insbes. bei global agierenden Unternehmen einfach und zügig Reichweite generiert werden kann (\#5). Zugleich bedarf es der Vertrautheit, d.h. der Möglichkeit, Themen ein Gesicht zu geben, und der Nutzung von Face-to-Face-Kommunikation gerade in Veränderungsprozessen, da Inhalte im Rahmen des persönlichen Austauschs besser im Gedächtnis bleiben. Es gehe darum, „durch eine sehr persönliche Ansprache wirklich auch eine Veränderung im Denken anzustoßen“, so die Kommunikatorin der Deutschen Bahn (\#2). Demzufolge stehen wie in der theoretischen Fundierung bereits dargelegt ein offener und bedürfnisgerechter Austausch orientiert an einem hohen $\mathrm{Ma} \beta$ an Empathie im Vordergrund (Deutinger 2017, S. 10; Mast und Fiedler 2007, S. 567).

In der Verbindung von persönlicher und medienvermittelter Kommunikation wird das gesamte Set an Kommunikationsmitteln, -kanälen und -plattformen, welches der internen Kommunikation zur Verfügung steht, genutzt sowohl im Rahmen klassischer Regelformate als auch anlassbezogener, eigens für die interne Kommunikation von Nachhaltigkeitsthemen konzipierter Sonderformate. Als Regelformate werden v.a. Town Halls, Workshops, Trainings und Schulungen sowie Meetings genannt, bei Printbzw. digitalen Formaten etwa Mitarbeitermagazine oder Flyer sowie (Social-)Intranet, Mitarbeiter-App, Newsletter und Mitarbeiter-TV. Vereinzelt kommen Blogs, Podcasts, Web-Trainings und Führungskräfte-Plattformen für die Veränderungskommunikation zum Einsatz. Komplettiert wird 
die Klaviatur durch externe soziale Medien wie Instagram, Facebook und YouTube, die (eher jüngere) Mitarbeiter ebenfalls erreichen.

Ein Fokus liegt in diesem Zusammenhang auf Kampagnenformaten - ein Ergebnis der Befragung, das im Vergleich mit anderen Strategie- und Veränderungsprozessen gerade bei Nachhaltigkeit besonderes Gewicht zu haben scheint. Kampagnen sind zeitlich befristete, thematisch begrenzte und dramaturgisch angelegte kommunikative Strategien mit dem Ziel, Aufmerksamkeit zu erzeugen (Röttger 2009, S. 9). Sie weisen durch aktuelle, relevante Bezüge eine hohe Sichtbarkeit auf, wie etwa die Bayer-Kampagne „\#voranbringen“ (\#1) und die „Das ist grün.“-Kampagne der Deutschen Bahn (\#2). Ergänzt werden sie durch themenspezifische, aktuelle Sonderformate wie z.B. das interne „Nachhaltigkeitsfrühstück 2020+“ von Kärcher (\#5), das als interaktiver und internationaler Workshop mit Vorstandsbeteiligung zum Thema Nachhaltigkeit durchgeführt wurde, oder die „Go-See-Share-Reisen“ von Nestlé, bei denen u.a. auch Mitarbeitende Produktionsstätten oder Herkunftsländer von Rohstoffen besuchen und erleben können, wie das Nachhaltigkeitsengagement vor Ort umgesetzt wird. Ziel dieser Kampagnen ist es, Mitarbeitende zu informieren und sie zu Multiplikatoren werden zu lassen.

Mit Blick auf die Aufbereitung der Kommunikation unter kommunikationstechnischen, inhaltlichen und sprachlichen Gesichtspunkten stimmen die Befragten überein, dass vornehmlich auf klassische Kommunikationstechniken zurückgegriffen wird. Dazu zählen unter anderen (Erklär-)Texte, Videos, Kurzfilme, (Experten-)Interviews, Grafiken und Schaubilder. Die Kommunikationstechnik des Storytellings wird in den Interviews nicht namentlich genannt, aber kommt implizit zum Ausdruck, wenn Personen im Rahmen der internen Kommunikation von ihrem Alltag erzählen und so implizit Nachhaltigkeitsbotschaften vermitteln (\#2). Die große Bedeutung, die der emotionalen Change-Story in der Theorie zugeschrieben wird, um Inhalte in die Verstehenswelt der Mitarbeitenden zu transferieren (Wagner 2019, S. 89; Mast 2016, S. 476), lässt sich durch die Interviews somit nur teilweise belegen. In inhaltlicher Hinsicht ist es Kernaufgabe der Kommunikation, komplexe Inhalte verständlich zu erklären, sie auf die unterschiedlichen Unternehmensbereiche und den Arbeitsalltag der Mitarbeitenden zuzuschneiden und mit Beispielen zu versehen, sodass die Komplexität der Nachhaltigkeitsthematik reduziert wird (\#2, \#3, \#5). Hier bestätigt sich die Annahme, dass Nachhaltigkeit als mehrdimensionales Thema besondere Anforderungen an das Themenmanagement stellt (Mast und Fiedler 2007, S. 569f.). Dazu gehöre auch eine verständliche, nachvollziehbare sowie prägnante Sprache zum „Sehen und Anfassen“ betont der befragte Kommunikationsexperte des Münchner Flughafens (\#3). Hierbei spielt auch der Erlebnis-Faktor, der in Veränderungsprozessen oft empfohlen wird (Mast und Fiedler 2007, S. 567) eine entscheidende Rolle. Jedoch ist diesbezüglich ein minutiöses Austarieren von Unterhaltung und Glaubwürdigkeit notwendig, hält eine Mitarbeiterin der Kommunikation von McDonald's (\#6) fest. Damit nimmt sie Bezug auf unterschiedliche Kommunikationsmodi (Lünenborg 2005, S. 126, 159; Huck-Sandhu 2014, S. 655 f.), deutet aber hinsichtlich der Tonalität auch die Dichotomie zwischen der eher objektiven, informationsorientierten Vermittlung von Fakten und den stärker emotionalen Komponenten im persönlichen Gespräch an. In den sozialen Medien wird auch im Innenverhältnis auf einen ,unterhaltsamen“ Stil gesetzt (\#6).

Hinsichtlich Emotionen lässt sich beobachten: Die Befragten stimmen einerseits darin überein, dass die Gesamtdebatte um Nachhaltigkeit nicht emotionalisiert werden dürfe, da Emotionalisierung häufig als „Polarisieren“ wahrgenommen werde. So betont etwa einer der Kommunikatoren (\#8), dass ein Spannungsfeld zwischen Emotionalisierung auf der einen Seite und Glaubwürdigkeit auf der anderen Seite bestehen könne. Andererseits ist der Rückgriff auf emotionale Komponenten insbesondere für die Verinnerlichung von Fakten auf der Ebene der Mitarbeitenden essenziell (\#6, \#9). Gefragt sei somit eine „offene, ehrliche, authentische Kommunikation auf Augenhöhe“ auf einem sachlich-informativen Fundament (\#7). Nur auf diesem Wege ist es möglich das in der Literatur umschriebene erfolgskritische Maß an Glaubwürdigkeit zu erzielen (Weder und Karmasin 2015, S. 939).

Die Grundhaltung, die in den Gesprächen deutlich wird, kommt dem theoretischen Konstrukt des Sensegivings (Weick 1995; Zahn et al. 2008) sehr nahe. In operativer Hinsicht spiegeln hierbei, ebenso wie bei der internen Veränderungskommunikation insgesamt, Kommunikationsdichte, -fülle, Stetigkeit und Konstanz sowie Glaubwürdigkeit, Anspruch und Überzeugungskraft eine Rolle. In den Gesprächen (\#6, \#7, \#9) zeigt sich zudem die Notwendigkeit einer Dialogorientierung - eine in der Literatur zur internen Nachhaltigkeitskommunikation vielfach als essenziell gehandelte Eigenschaft der Kommunikation. Die auf die Strategieimplementierung abzielende interne Kommunikation erfolgt zwar in den befragten Unternehmen mehrheitlich nach dem Top-down-Prinzip, jedoch kommt auch der Bottom-up-Kommunikation und dem informellen Austausch im Sinne horizontaler und vertikaler Kommunikation eine wichtige Rolle zu. Zusammenfassend lässt sich festhalten: Die Bedeutung und Rolle, die der internen Nachhaltigkeitskommunikation im Rahmen strategischer Transformation in der Literatur zugeschrieben wird, findet ihre Entsprechung in einer ganzheitlich angelegten, breiten internen Kommunikation, die auch Schnittstellen zur externen Kommunikation einbindet und unter kommunikationstechnischen, inhaltlichen und sprachlichen Gesichtspunkten 
eine große Vielfalt an Ausgestaltungsmöglichkeiten aufweist.

Durchweg wird in den Leitfadeninterviews deutlich: Interne Kommunikation kann Anstöße geben, aber eine Steuerung von Wahrnehmung, Einstellungen und Verhalten ist auch im Bereich der Veränderungskommunikation nicht möglich. Neben der aktiven Kommunikation über interne Medien und Kanäle werden hierfür spezifische Informationsmaterialien für Führungskräfte entwickelt, Trainings und Schulungen eingesetzt und Angebote zur Reflexion des eigenen Verhaltens geboten. Der Idealfall ist die „Verinnerlichung" der Nachhaltigkeitsthematik (\#9). Somit sind die in der Literatur beschriebenen Ziele und Aufgabenfelder von Veränderungskommunikation auch im Bereich der internen Nachhaltigkeitskommunikation wiederzuerkennen.

\subsubsection{FF4: Inwiefern tragen kulturelle und institutionelle Parameter zur kognitiven und affektiven Verankerung sowie zur Operationalisierung der Strategie bei?}

In der Literatur liegt ein besonderes Augenmerk auf der Institutionalisierung der strategischen Neuausrichtung und der Implementierung von Strategie durch die direkte Wirkung der internen Kommunikation. In den Interviews bestätigt sich, dass die Unternehmenswerte eine erste relevante Größe darstellen. Diese sind in den Unternehmen der Befragten strukturell fest verankert. Was bei Bayer (\#1) die Mission ist, findet sich bei Schnellecke (\#9) im „Spirit“, bei Unternehmen A (\#8) im übergeordneten „Purpose“ und beim Flughafen München (\#3) im Markenkern wieder. Darin sind auch die Themen Nachhaltigkeit und unternehmerische Verantwortung als Selbstverpflichtung festgehalten, so dass den Unternehmenswerten auch bei der der strategischen Neuausrichtung und bei der Strategieimplementierung eine wesentliche Rolle zukommt. Sie stellen Basisprinzipien des Handelns dar, die als Eckpfeiler der internen Kommunikationsarbeit wirken und dem Nachhaltigkeitsengagement Glaubwürdigkeit verleihen.

Für das Verhältnis zwischen den Unternehmenswerten und den Mitarbeitenden lassen sich in der Befragung zwei Tendenzen ausmachen: Während die Unternehmenswerte etwa bei der Ford Deutschland Werke GmbH (\#4) einen eher normativen Charakter besitzen und als unternehmensweit bekannte Richtlinien für das Verhalten von Mitarbeitenden fungieren und umgesetzt werden müssen (verbindliche Handlungsprinzipien), werden die Werte in den anderen Unternehmen als übergeordnete Orientierungspunkte für Grundhaltungen und Einstellungen der Mitarbeitenden verstanden und haben eher Aufforderungscharakter. Unabhängig von der Verbindlichkeit der beiden Perspektiven: Alle Befragten sehen die Unternehmenswerte als inhärenten Teil der Unternehmenskultur, die von Kulturfaktoren auf fundamentale Art und Weise geprägt werden. Somit lassen sich die Hinweise aus der Literatur bestärken. Auch herrscht Einigkeit darüber, dass die Unternehmenskultur im Rahmen der strategischen Neuausrichtung eine zentrale Rolle spielt. Kultur wird als bestehende ,große Struktur“ (\#5) beschrieben, in die sich das Nachhaltigkeitsengagement der Mitarbeitenden einbetten muss und innerhalb deren Rahmenvorgaben sich die interne Veränderungskommunikation entfaltet. In der Zusammenschau aller Aussagen der Befragten lässt sich schlussfolgern, dass die Unternehmenskultur eine unterstützende Funktion bei der Vermittlung der Nachhaltigkeitsthematik einnimmt, diese als solche aber noch nicht mehrheitlich wahrgenommen und aktiv genutzt wird. Auch hier lässt sich eine Parallele zur bereits identifizierten impliziten Vermittlung des Wandels über die Werte und die Unternehmenskultur ziehen.

Eine entscheidende Rolle für die strategische Neuausrichtung und die operative Strategieimplementierung haben die Führungskräfte. Dies geht deutlich aus den Aussagen der Befragten hervor. Auch Führungskräfte hat die interne Kommunikation über die speziell für sie vorgesehenen Kanäle im Veränderungsprozess abzuholen und einzubinden, so die einhellige Überzeugung. Sie müssten die Relevanz der Thematik erkennen, ihr Bewusstsein schärfen und ausreichend informiert sein - kurz gesagt: befähigt werden - über die Informationskaskade den „Nachhaltigkeitsgedanken in die Teams zu tragen“ (\#3). Führungskräfte werden auch als „Transmissionsriemen“ zwischen dem Vorstand und der breiten Belegschaft beschrieben. Sie sind lebendiges Vorbild, Meinungsführer, Multiplikator und Dialogpartner - Rollenverständnisse, die sich auch in der Literatur so finden (Mast und Huck 2008; Buchholz und Knorre 2019; Dunphy et al. 2007, S. 217). Zudem sei es Aufgabe der Führungskräfte, Feedback einzuholen und die gewonnenen Informationen an den Vorstand zurückzuspiegeln, also den Bottom-up-Informationsfluss zu sichern (\#1).

Bei Nestlé (\#7) kommen neben Führungskräften auch interne „Nachhaltigkeits-Champions“ zum Einsatz. Sie vertreten das Thema Nachhaltigkeit für jedes Business und bilden ein Corporate Volunteering-, CSR- und/oder Nachhaltigkeitsnetzwerk. Durch diese Gruppe von internen Multiplikatoren wird ein schnellerer und umfassenderer Informationsaustausch möglich. Auch die Informationsverarbeitung wird durch das persönliche Gespräch mit einem solchen Experten verbessert. Blickt man zurück in die Literatur, so lassen sich Nestlés "Nachhaltigkeits-Champions“ (\#7) als Change Agents bzw. Promotoren im Innovationsprozess bezeichnen (Dunphy et al. 2007; Göll et al. 2019, S. 37; Allen 2016). 


\subsubsection{FF5: Worin bestehen die zentralen Herausforderungen bei der Strategieimplementierung durch Kommunikation aus Sicht der Kommunikatoren?}

Basierend auf den Aussagen der befragten Kommunikationsfachleute kann festgehalten werden, dass die interne Kommunikation bei der Nachhaltigkeitsthematik mit dem Ziel der Strategieimplementierung mit einer Reihe von Herausforderungen konfrontiert wird. Nicht nur die Mitarbeitenden selbst, auch die unternehmerischen Rahmenbedingungen und Nachhaltigkeit als besonders fordernder Kommunikationsinhalt können die erfolgreiche Realisation der strategischen Neuausrichtung verhindern. Aus Sicht der Kommunikation liegt die Problematik insbesondere darin, dass nicht alle Herausforderungen kommunikativ überwunden werden können. Die Veränderung anderer Einflussgrößen erscheint unumgänglich.

Am häufigsten wird die Komplexität der Thematik genannt, die durch den Gesamtumfang und den kontextabhängigen Facettenreichtum sowohl die kommunikative Vermittlung als auch den Verstehens- und Sinnbildungsprozess der Mitarbeitenden erschweren (\#1, \#3, \#5, \#10). Je heterogener die Kommunikationszielgruppe, je diverser Rezeptionsgewohnheiten und je unterschiedlicher die Erwartungen und Ansprüche an Inhalte und Aufbereitung, desto größer die Herausforderungen gerade im Veränderungsprozess. Auch die Aufrechterhaltung einer effizienten Kommunikationskaskade, insbesondere in der Vermittlung über die Führungskräfte, die inhaltliche Priorisierung und der Umgang mit den Ressourcen Zeit und Aufmerksamkeit werden als Herausforderung angesehen (\#7). Hinzu kommt eine in vielen Fällen anfänglich skeptische Grundhaltung der Mitarbeitenden gegenüber der Nachhaltigkeitsthematik, so dass ein erhöhtes $\mathrm{Ma} ß$ an kommunikativer Begleitung solcher Veränderungsprozesse nötig ist. Auch der in der Literatur genannte schwerwiegendste Hinderungsgrund bei der Operationalisierung von Nachhaltigkeitsstrategien tritt in den Interviews zu Tage: der unter Mitarbeitenden weit verbreitete Mangel an Veränderungsbereitschaft (Zahn et al. 2008, S. 25; Krüger 2014, S. 19f.). Eine Kommunikationsverantwortliche bei McDonald's (\#6) etwa betont: „Menschen sind Gewohnheitstiere: Nur nicht das eigene Verhalten ändern". Somit wird die Notwendigkeit von Wissens-, Einstellungs- und Verhaltensänderungen zur Strategieimplementierung (Raps 2017, S. 27) auch vom praktischen Standpunkt bestätigt.

\subsection{Diskussion}

Betrachtet man die Ergebnisse aus den Leitfadengesprächen, so bestätigen sich die wesentlichen Grundannahmen und -erkenntnisse, die im Forschungsfeld zur allgemeinen internen Veränderungskommunikation bis dato vorliegen. Die Kernprinzipien scheinen - wenig überraschend - auch für nachhaltigkeitsbezogene Strategie- und Veränderungsprozesse zu gelten. Allerdings ergaben die Interviews auch einige neue Befunde, die spezifisch mit der Etablierung einer neuen Nachhaltigkeitsstrategie verbunden zu sein scheinen - und zeigten ein Ergebnis, das dem Stand der Literatur sogar widerspricht.

Für die Diskussion und Einordnung der Ergebnisse bedarf es zunächst eines Blickes auf die Limitationen: Durch den qualitativen Charakter der Studie war es möglich, die Praxis interner Veränderungskommunikation explorativ und unternehmensspezifisch, mit Blick auf die Ausrichtung, den Umfang und Kontext des jeweiligen Strategieprozesses zu erheben. Qualitative Forschung bringt aber auch klare Limitationen mit sich. Dazu gehören v.a. methodenspezifische Aspekte wie z.B. die Tatsache, dass sich unterschiedliche Interviewpartner in den individuellen Gesprächssituationen dem Forscher gegenüber in unterschiedlichem Maße öffnen (Helfferich 2019, S. 672) und die Interviewführung das Gespräch, trotz aller Offenheit, mit Fragen und Nachfragen lenkt (Kaiser 2014, S. 33). Hinzu kommt die mangelnde Verallgemeinerbarkeit der Aussagen. Für die hier vorliegende Studie mag zudem die Teilnahmequote der angefragten Unternehmen klein erscheinen. Trotz der begrenzten Teilnahmebereitschaft unter den 96 angefragten Unternehmen war die Fallzahl am Ende groß genug, um die erforderliche Unterschiedlichkeit der Fälle sicherzustellen und zugleich klein genug, um eine „,deep, case-oriented analysis“ (Sandelowski 1995, S. 183) im qualitativen Zugang zu ermöglichen. Als Gründe für die Zurückhaltung der Praxis kann vermutet werden, dass die Auskunftsbereitschaft zu Strategiethemen per se geringer ist. Zudem ist die hier untersuchte Fragestellung voraussetzungsvoll und neuartig. Mit der Verbindung von interner Kommunikation, Veränderungskommunikation und Nachhaltigkeitsstrategie ist sie an der Schnittstelle zwischen verschiedenen Arbeitsfeldern und Abteilungen angesiedelt. Zudem kann vermutet werden, dass manche angefragte Kommunikationsexpert*innen sich in der Verbindung der Themenfelder ggf. nicht fachkundig genug fühlten, um teilzunehmen. Durch die Homogenität der Befragtengruppe bezüglich der in Abschn. 3.1 genannten Auswahlkriterien in Verbindung mit der Heterogenität der jeweiligen Strategieprozesse und -phasen liegt mit den zehn Interviews nichtsdestotrotz ein vertiefender und zugleich breiter Einblick in die interne Veränderungskommunikation bei der Etablierung von Nachhaltigkeitsstrategien in Groß- und großen mittelständigen Unternehmen vor.

Wie auf Grundlage der Literatur zu erwarten war, schreiben die Befragten den Mitarbeitenden (und in der Folge auch der internen Kommunikation) eine wichtige Rolle für die Gestaltung des Veränderungsprozesses zu. Kernaufga- 
ben der Veränderungskommunikation liegen in der Information, der Integration, der Motivation und der Aktivierung von Mitarbeitenden, um die angestrebte Wissens-, Einstellungs- und Verhaltensakzeptanz bei Mitarbeitenden zu fördern - klassische Ziele, die für Veränderungsprozesse jeder Art gelten (vgl. Abschn. 2.2; Krüger 2014, S. 19; Deutinger 2017, S. 10; Zowislo-Grünewald und Schwab 2003, S. 7). Die Kernergebnisse können entlang der drei kommunikativen Wirkungsebenen zusammenfasst werden: Auf der kognitiven Ebene wird das Ziel der Information und der Steigerung des Wissenstandes der Mitarbeitenden durch den vermehrten Einsatz von informativen und faktenbasierten Formaten verfolgt. Das Ziel des Commitments, der Aktivierung und damit der affektiven Verankerung wird verstärkt über emotionale, auch unterhaltende, immer aber auf Beteiligung und persönlichem Austausch beruhenden Kommunikationsformate umgesetzt. Die konative Verankerung der Nachhaltigkeitsorientierung wird durch die Nutzung verschiedener Kommunikationsmodi und die Vorbild- und Multiplikatorfunktion der Führungskräfte begünstigt, die auch in der Literatur stark betont wird. Die sukzessive Kommunikation von Teilaspekten, die kontinuierliche Wiederholung sowie die Omnipräsenz des Themas und der angestrebten Verhaltensweisen in den internen Medien sind darauf ausgelegt, dass Nachhaltigkeit im Unternehmensalltag akzeptiert und gelebt wird. Unternehmenswerte, Unternehmenskultur und Führungsverhalten bildet das Fundament, auf dem interne Veränderungskommunikation aufsetzen kann. Kommunikation begleitet, verstärkt und vermittelt, was im Selbstverständnis des Unternehmens angelegt und durch den Strategieprozess vertieft oder weiterentwickelt werden soll.

Während diese Erkenntnisse konform zum Stand der Literatur sind, überrascht eines der in Abschn. 3.2 beschriebenen Ergebnisse: Der narrative Modus - der in der Literatur in Form des Storytellings als essenziell für die Veränderungskommunikation erachtet wird (vgl. Abschn. 2.3) scheint im nachhaltigkeitsorientierten Strategieprozess der befragten Unternehmen kaum eine Rolle zu spielen:

- Folgt man der Literatur und Studien zur internen Kommunikation in Strategie- und Veränderungsprozessen allgemein, so gelingt die Aktivierung der Mitarbeitenden über narrative Ansätze, v.a. über die emotionalisierende Technik des Storytellings, besonders gut (Deutinger 2016, S. 100; Wagner 2019, S. 89). Über Anwendungsund Erfolgsgeschichten können (neue) Werte und Verhaltensweisen intuitiv vermittelt werden. Sie schaffen Nähe, Glaubwürdigkeit und Gemeinschaft. Zudem ermöglicht Storytelling, wünschenswerte Ziele und Zustände, die in der Zukunft liegen, vorweg verständlich und motivierend anzudeuten. Allesamt Vorteile, die in Strategie- und Veränderungsprozessen sowie im Rahmen der Werte- und Kulturvermittlung in Unternehmen in der Regel inten- siv genutzt werden (Huck-Sandhu 2014, S. 661). Dass die Leitfadengespräche dies nicht stützen, sondern den narrativen Modus der internen Kommunikation sogar als wenig oder nicht relevant einstufen, widerspricht der Literatur und empirischen Erkenntnissen der allgemeinen Strategiekommunikation. Eine mögliche Erklärung könnte darin liegen, dass Storytelling als Technik im Bereich der Nachhaltigkeitskommunikation so weit verbreitet ist, dass die Befragten diesem Thema im Veränderungsprozess keine gesteigerte Bedeutung zuweisen wie sie in qualitativen und quantitativen Studien zur internen Kommunikation i. A. sowie zur Veränderungskommunikation zu allgemeinen Strategiethemen in den letzten Jahren als Trendthema an Bedeutung gewonnen hat. Anders formuliert: Storytelling und mit ihm narrative Kommunikation insgesamt könnten in der Nachhaltigkeitskommunikation und in den befragten Kommunikationsabteilungen mittlerweile so selbstverständlich sein, dass dieser Technik kein besonderes Augenmerk mehr gilt. Die Interviews haben allerdings gezeigt, dass Personalisierung als verwandte Technik durchaus eingesetzt wird. Eine alternative Erklärung könnte demnach sein, dass Nachhaltigkeit als normatives Thema (Prexl 2010, S. 40) per se bereits emotional besetzt ist. In diesem spezifischen Themenfeld wäre dann eine weitere Emotionalisierung, wie sie durch Storytelling erreicht werden soll, unnötig, ggf. sogar kontraproduktiv. Diejenigen Mitarbeitenden, die Nachhaltigkeit als sinnvoll im privaten und beruflichen Handeln akzeptieren, bedürfen keiner zusätzlichen emotionalen Aktivierung. Mitarbeitende, die es noch zu überzeugen gilt, sind im Hinblick auf die angestrebte Einstellungsveränderung zunächst stärker kognitiv, also im Hinblick auf Information und Erklärung, einzubinden. Das Ergebnis, dass die befragten Kommunikationsexpert*innen der informierend-rahmenden Kommunikation, also dem erklärenden Modus (Lünenborg 2005, S. 126, 159) eine wichtige Rolle zuschreiben, scheint diese These zu stützen. Die weiterführende Forschung kann hier ansetzen, indem sie neben einer breiteren Analyse zu narrativer Kommunikation verstärkt Hintergründe, Ausformung und Rezeption und Wirkung analysiert. Über einen experimentellen Ansatz könnte beleuchtet werden, ob die Prämisse der hier befragten Kommunikatoren sich auch in der Wahrnehmung von Storytelling-Formaten durch Mitarbeitende bestätigt.

Zwei weitere Befunde, die hervorgehoben werden können, sind die besondere Wichtigkeit des erklärenden Modus und die Fokussierung vor allem auf persönliche und digitale Medien. Sie könnten sich durch den spezifischen Charakter des Themas Nachhaltigkeit erklären: 
- Die Interviews deuten darauf hin, dass dem erklärenden Modus (Lünenborg 2005, S. 126, 159) in der nachhaltigkeitsorientierten Veränderungskommunikation gesteigerte Bedeutung zuzukommen scheint. Das Ziel der angestrebten Wissens- und Einstellungsveränderung zugunsten Nachhaltigkeit, das maßgeblich auf der Informations- und Überzeugungsarbeit interner Veränderungskommunikation beruht, wird in nachhaltigkeitsorientierten Strategieveränderungen als besonders anspruchsvoll beschrieben. Nachhaltigkeit als Thema ist komplex und mehrdimensional (vgl. Abschn. 2.1), etwa indem Auswirkungen einer Verhaltensänderung auf die Zukunft gerichtet und abstrakt sein können. Nachhaltigkeitsorientierte Strategien sind somit besonders erklärungsbedürftig. Für die interne Veränderungskommunikation könnte dies bedeuten, dass sie deutlich stärker als interne Kommunikation i.A. und Veränderungskommunikation zu allgemeinen Strategieprozessen i.B. problematisieren, erklären, Hintergründe aufzeigen und den Nutzen der (Verhaltens-)Veränderung betonen sollte. Für das Forschungs- und Praxisfeld ließen sich Befunde aus der Innovationskommunikationsforschung (Zerfaß und HuckSandhu 2007, S. 853 ff.) fruchtbar machen: Auch Innovationen sind abstrakt, mehrdimensional, in die Zukunft gerichtet und können Ungewissheit, Ängste und Unsicherheiten auslösen. Deshalb sind sie - ganz ähnlich zu Themen im Zusammenhang mit Nachhaltigkeitsstrategien - erklärungsbedürftig und bedürfen einer intensiven Kommunikation (Fink und Grupe 2020, S. 4). Nachfolgestudien könnten gezielt den spezifischen Einsatz und die Ausgestaltung von Kommunikationsmodi im Kontext interner Veränderungs- und Nachhaltigkeitskommunikation untersuchen. Über Case Studies wäre ein vergleichender Ansatz, auch ergänzt um eine Materialanalyse möglich. Experimentelle Studien sowie quantitative Befragungen könnten Hinweise liefern, inwiefern das Thema Nachhaltigkeitsstrategien besondere Anforderungen an die Kommunikation - z. B. Wissensvermittlung, Einstellungs- und Verhaltensveränderungen - stellt und sich darin von anderen Strategiethemen unterscheiden könnte. Die Interviews deuten zudem darauf hin, dass der Sensibilisierungsphase bei Veränderungsprozessen $\mathrm{zu}$ Nachhaltigkeit mehr Gewicht zukommen könnte als in thematisch anders gelagerten Strategie- und Kulturwandelprozessen. Für Nachfolgestudien lässt sich zudem die Frage aufwerfen, ob je nach Prozessphase unterschiedliche Modi dominieren oder derselbe Modus je nach Phase unterschiedlich umgesetzt wird.

- Persönliche Kommunikation und digitale Medien werden von den Befragten als dominante Kanäle im Strategieprozess genannt. Auffallend ist somit, dass gedruckte Medien in den Interviews kaum genannt werden. In der Literatur wird den Printmedien wie z.B. der Mit- arbeiterzeitschrift als typischem Hintergrundmedium die Rolle der Information, Einordnung und Rahmung zugeschrieben. Betrachtet man die Praxis interner Unternehmenskommunikation ganz allgemein, so zeigen Längsschnittstudien unter den Top 500-Unternehmen in Deutschland zwar einen Aufwuchs digitaler Kanäle und eine gleichbleibend hohe Bedeutung der persönlichen Kommunikation für die Ansprache der Mitarbeitenden; ein allgemeiner Bedeutungsverlust der gedruckten Medien lässt sich jedoch nicht bestätigen, im Gegenteil: Printmedien bleiben in der internen Kommunikation weiter wichtig (Spachmann und Huck-Sandhu 2020). Als eine mögliche These für den starken Fokus auf die persönlichen und digitalen Medien in der internen Veränderungskommunikation lässt sich formulieren, dass nachhaltige Unternehmen klassische Printformate aus Gründen der Nachhaltigkeit weitgehend abgeschafft haben könnten. Eine andere mögliche These kann sein, dass auch in nachhaltigkeitsorientierten Unternehmen weiterhin Printmedien genutzt und verteilt werden, aber im Teilbereich interne Nachhaltigkeitskommunikation bewusst auf Papier, Druck und die physische Verteilung von Printmaterial verzichtet und stärker auf nachhaltige(re) Medien setzt. Wie Marshall McLuhan einst schrieb: „,The medium is the message“. Ergänzende Interviews könnten die Beweggründe für diese Ausblendung der Printkommunikation genauer untersuchen und zugleich bei Mitarbeitenden erheben, inwiefern der Medieneinsatz zu Nachhaltigkeitsthemen bewusst oder unterbewusst wahrgenommen wird.

Inwiefern sich insbesondere die neuen, von der Literatur teils abweichenden Befunde für die interne Veränderungs- und Nachhaltigkeitskommunikation in weiteren Studien bestätigen lassen, werden Nachfolgestudien zeigen müssen. Qualitative Folgestudien könnten Hinweise auf mögliche Erklärungen bieten. Quantitative Studien könnten dazu beitragen, die Ergebnisse im größeren Maßstab zu prüfen und systematische Zusammenhänge z.B. mit kulturellen und institutionellen Rahmenfaktoren aufzudecken. Zudem könnte mit einer größeren Stichprobe und einer quantitativ ausgerichteten Anlage geprüft werden, ob und wenn ja, wo sich systematische Zusammenhänge mit Kontextvariablen zeigen. Bei der Auswahl der Grundgesamtheit (vgl. Abschn. 3.1) wurde die Branchenzugehörigkeit als untergeordnetes Kriterium eingestuft - eine Setzung, die im Rahmen der qualitativen Analyse bestätigt werden konnte. Zusammenhänge zwischen der Herangehensweise an die interne Nachhaltigkeits- und Veränderungskommunikation einerseits und Branche, Unternehmensgröße, Organisation der Kommunikation sowie anderen institutionellen Kriterien andererseits waren nicht erkennbar. Zwar ist anzunehmen, dass der Stellenwert des Nachhaltigkeitsge- 
dankens z. B. von Branche zu Branche variiert und Mitarbeitende demnach schon vorab in unterschiedlichem Maße für das Thema sensibilisiert sind. Jedoch werden auch bei der Implementierung der neuen strategischen Ausrichtung in nachhaltigkeitsnahen Branchen dieselben Ziele verfolgt, ähnliche Kommunikationsmaßnahmen initiiert und damit Entwicklungen angestoßen.

\section{Zusammenfassung und Implikationen für die Praxis}

Das gesellschaftliche, politische und wirtschaftliche Umfeld, in dem Unternehmen heute agieren, ist komplexer und volatiler geworden (Mast 2008, S. 2). Wo in Unternehmen Veränderungen auftreten, bedarf es der internen Veränderungskommunikation. Im Falle einer strategischen Neuausrichtung des Unternehmens in Richtung unternehmerische Nachhaltigkeit und Verantwortung sieht sich die interne Nachhaltigkeitskommunikation in dieser Rolle und damit teils großen Herausforderungen gegenüber. Sie hat dann nicht nur einen wesentlichen Beitrag zur Veränderung der unternehmerischen Rahmenbedingungen, sondern v.a. auch zur Gewinnung der Zustimmung der Mitarbeitenden für den eingeschlagenen Weg und ihre Bereitschaft zur Mitwirkung zu leisten (Klasen 2019, S. 164).

Im Mittelpunkt des Beitrags stand die Frage, welche Rolle die interne Veränderungskommunikation für die Etablierung von Nachhaltigkeitsstrategien in Unternehmen spielen kann. Neben der Rolle und Reichweite einer solchen Veränderungskommunikation wurden Rahmenbedingungen und Erfolgsfaktoren für eine solche Kommunikation mit Mitarbeitenden untersucht. ,[C]apturing the hearts and minds of all employees“ (Dunphy et al. 2007, S. 93) erfolgt bei nachhaltigkeitsorientierten Strategieveränderungen in weiten Teilen ähnlich zu Veränderungskommunikation und -management für allgemeine Strategieprozesse. Im Rahmen der Erhebung wurde deutlich, dass Veränderungskommunikation in den Nachhaltigkeitsprojekten der befragten Unternehmen zwar nicht zwingend als solche bezeichnet, aber in weiten Teilen konform zum Stand der Literatur umgesetzt wird. Unterschiede zeigten sich bezüglich der Rolle des narrativen und des erklärenden Kommunikationsmodus sowie beim Medieneinsatz.

Für die Praxis lässt sich ableiten, dass interne Kommunikation systematisch eingebunden, strategisch angelegt und strukturiert umgesetzt sein muss. Die dargelegten Herangehensweisen und der hohe Professionalisierungsgrad, der in den befragten Unternehmen bei der Kommunikationsstrategie, beim Medieneinsatz und der Umsetzung der Formate deutlich wird, bildet eine wesentliche Basis für die Strategieimplementierung. Festgemacht werden kann dies u.a. am intensiven Rückgriff auf die persönliche Kommunikation, an der Gewichtung von Beteiligung und Befähigung der Mitarbeitenden sowie von Glaubwürdigkeit und am bewussten Einsatz verschiedener Kommunikationsmodi. Zugleich ist klar: Die interne Nachhaltigkeitskommunikation ist allein nicht imstande, einen Wandel zu bewirken. Erst im Zusammenspiel mit anderen Faktoren kann sie einen wesentlichen Beitrag zur Etablierung neuer Nachhaltigkeitsstrategien bei Mitarbeitenden leisten. 


\section{Anhang}

\section{Anhang A1: Interviewleitfaden}

\section{Interne Nachhaltigkeits- und \\ Veränderungskommunikation}

\section{Allgemeine Fragen}

- Können Sie ganz kurz umreißen, was Ihre Funktion und Ihre Aufgaben im Unternehmen sind?

- Worin besteht das Nachhaltigkeitsengagement Ihres Unternehmens (Startzeitpunkt, Ziele, Inhalte)?

- Inwieweit wird die Integration dieses Engagements in die Unternehmensstrategie und damit in die Geschäftstätigkeit als Veränderungsvorhaben behandelt?

- Wie ist die Veränderungskommunikation innerhalb ihres Unternehmens angelegt? Wo liegen Verantwortlichkeiten?

- Wer ist für die Kommunikation das Thema Nachhaltigkeit verantwortlich?

\section{Interne Nachhaltigkeitskommunikation als Instrument im Strategieprozess}

Bedeutung und Ziele

- Welche Rolle spielt die interne Kommunikation in Bezug auf Nachhaltigkeit (im Veränderungsprozess) in Ihrem Unternehmen?

- Gibt es konkrete Ziele, die Ihr Unternehmen bei der internen Kommunikation zu diesen Themen verfolgt?

- Worin liegen die zentralen Aufgaben und Funktionen der Nachhaltigkeitskommunikation (im Veränderungsprozess)?

Kommunikation

- Wie genau wir das Thema Nachhaltigkeit als Teil einer neuen Strategie intern vermittelt und verständlich gemacht?

- Auf welche Art und Weise wird das Thema von Mitarbeitenden aufgenommen und verarbeitet?

- Wie stellen sie sicher, dass Mitarbeitende das Engagement und ihre persönliche Beteiligung daran als sinnvoll erachten?

- Was aktiviert und motiviert Mitarbeitende, sich mit dem Thema auseinanderzusetzen und es umzusetzen?

- Was hindert Mitarbeitende daran Nachhaltigkeit umzusetzen?

- Welchen Herausforderungen begegnen Sie bei der Vermittlung der Themen an Mitarbeitende?

- Inwiefern ist Nachhaltigkeit für Ihre Mitarbeitenden ein (relevantes) Thema in der informellen Kommunikation?

\section{Strategieimplementierung durch interne Nachhaltigkeitskommunikation}

Die Umsetzung von Nachhaltigkeit kommunikativ initiieren

- Welche Rolle nehmen Mitarbeitende bei strategischen Neuausrichtungen ein?

- Welchen Einfluss hat die interne Nachhaltigkeitskommunikation auf die Umsetzung der neuen Strategie durch Mitarbeitende?

- Wie gelingt es, dass Mitarbeitende die Nachhaltigkeitsstrategie in ihrem Arbeitsalltag umsetzen?

- Reichen kommunikative Maßnahmen aus, um Mitarbeitende über die neue Strategie zu informieren und davon zu überzeugen? Wenn nein, was sind unterstützende Konzepte oder Methoden?

Einflussfaktoren

- Wie schaffen Sie Verständnis, Akzeptanz und Begeisterung/Commitment für die Nachhaltigkeitsstrategie und deren Umsetzung?

- Welche Rolle spielen die Unternehmenswerte und die Unternehmenskultur bei der Verankerung der Strategie?

- Welche Rolle nehmen Führungskräfte und der Führungsstil bei der Strategieimplementierung ein?

Ausgestaltung

- Auf welchen Kanälen wird die Nachhaltigkeitsstrategie kommuniziert?

- Wie werden Mitarbeitende beteiligt?

- Wie schätzen Sie die Tonalität der Kommunikation ein? Rein informativ, erklärend, emotionsgeladen, argumentativ, werbend?

- Verändert sich die Kommunikationsintensität im Verlauf des Veränderungsprozesses/der Implementierung?

Praktische Erfahrungen

- Welche Erfahrungen haben Sie in Bezug auf die interne Kommunikation der neuen Nachhaltigkeitsstrategie bisher gemacht?

- Angesichts der immer lauter werdenden Forderungen nach Nachhaltigkeitskommunikation - wie schätzen Sie die Entwicklung der Aufgabe der Kommunikation auf diesem Feld in Zukunft ein?

\section{Schluss}

Gibt es noch etwas, das Sie zu diesem Thema gerne erzählen möchten? Etwas, das ihnen wichtig ist und bisher noch nicht angesprochen wurde? 


\section{Anhang A2}

Tab. 2 Deduktiver Kodierleitfaden

\begin{tabular}{|c|c|c|c|}
\hline Kategorie & Definition & Beispiel aus Expert*inneninterview & Kodierregel \\
\hline $\begin{array}{l}\text { Bedeutung der } \\
\text { internen Kom- } \\
\text { munikation von } \\
\text { Nachhaltigkeit }\end{array}$ & $\begin{array}{l}\text { Gewicht, welches internen Kom- } \\
\text { munikationsmaßnahmen zum The- } \\
\text { ma Nachhaltigkeit zugeschrieben } \\
\text { wird, um dieses im Unternehmen } \\
\text { zu platzieren und Veränderungen } \\
\text { anzustoßen }\end{array}$ & $\begin{array}{l}\text { Beim Thema Nachhaltigkeit sind die Mit- } \\
\text { arbeiter von besonderer Bedeutung. In den } \\
\text { letzten Jahren ist diese gestiegen }\end{array}$ & $\begin{array}{l}\text { Rolle der internen Kommunikati- } \\
\text { on und der Mitarbeitenden bei der } \\
\text { strategischen Fokussierung auf } \\
\text { das Thema Nachhaltigkeit }\end{array}$ \\
\hline $\begin{array}{l}\text { Ziel der internen } \\
\text { Nachhaltigkeits- } \\
\text { kommunikation }\end{array}$ & $\begin{array}{l}\text { Ergebnis, welches durch die inter- } \\
\text { ne Kommunikation des Themas } \\
\text { Nachhaltigkeit angestrebt wird }\end{array}$ & $\begin{array}{l}\text { Mitarbeitende begeistern und dazu moti- } \\
\text { vieren, sich mit der Thematik auseinan- } \\
\text { derzusetzen. Bewusstsein und Verständnis } \\
\text { schaffen, informieren, sensibilisieren und zu } \\
\text { nachhaltigen Verhaltensweisen befähigen }\end{array}$ & $\begin{array}{l}\text { Konkrete Wirkung, die gezielt } \\
\text { eingesetzte Kommunikations- } \\
\text { maßnahmen bei Mitarbeitenden } \\
\text { auslösen sollen }\end{array}$ \\
\hline $\begin{array}{l}\text { Rolle der Mitar- } \\
\text { beitenden bei der } \\
\text { strategischen Neu- } \\
\text { ausrichtung/der } \\
\text { Veränderung }\end{array}$ & $\begin{array}{l}\text { Funktion von Mitarbeitenden } \\
\text { bei der Implementierung einer } \\
\text { strategischen Neuausrichtung/ } \\
\text { eines Wandels }\end{array}$ & $\begin{array}{l}\text { Mitarbeiter erfüllen die Programme mit } \\
\text { Leben. Es dreht sich alles um sie, denn die } \\
\text { gesamte Strategie wurde um sie herum ent- } \\
\text { wickelt. Insofern sind sie der Dreh- und } \\
\text { Angelpunkt der ganzen strategischen Neu- } \\
\text { ausrichtung }\end{array}$ & $\begin{array}{l}\text { Aspekte, die die Bedeutung von } \\
\text { Mitarbeitenden bei der Implemen- } \\
\text { tierung neuer Strategien hervorhe- } \\
\text { ben }\end{array}$ \\
\hline $\begin{array}{l}\text { Inhaltliche Auf- } \\
\text { bereitung der } \\
\text { Nachhaltigkeits- } \\
\text { themen }\end{array}$ & $\begin{array}{l}\text { Art und Weise, wie Inhalte rund } \\
\text { um das Thema Nachhaltigkeit } \\
\text { an Mitarbeitende weitergegeben } \\
\text { werden }\end{array}$ & $\begin{array}{l}\text { Übersetzung und Transfer von der komple- } \\
\text { xen, fachlichen Ebene für die breite Beleg- } \\
\text { schaft: interessante, informative, überzeu- } \\
\text { gende und verständliche Aufbereitung }\end{array}$ & $\begin{array}{l}\text { Modi, Methoden und Vorgehens- } \\
\text { weisen, die dabei unterstützen, die } \\
\text { komplexen Inhalte zum Thema } \\
\text { Nachhaltigkeit für Mitarbeiten- } \\
\text { de verständlich und greifbar zu } \\
\text { machen }\end{array}$ \\
\hline
\end{tabular}


Funding Open Access funding enabled and organized by Projekt DEAL.

Interessenkonflikt L. Strottner und S. Huck-Sandhu geben an, dass kein Interessenkonflikt besteht.

Open Access Dieser Artikel wird unter der Creative Commons Namensnennung 4.0 International Lizenz veröffentlicht, welche die Nutzung, Vervielfältigung, Bearbeitung, Verbreitung und Wiedergabe in jeglichem Medium und Format erlaubt, sofern Sie den/die ursprünglichen Autor(en) und die Quelle ordnungsgemäß nennen, einen Link zur Creative Commons Lizenz beifügen und angeben, ob Änderungen vorgenommen wurden.

Die in diesem Artikel enthaltenen Bilder und sonstiges Drittmaterial unterliegen ebenfalls der genannten Creative Commons Lizenz, sofern sich aus der Abbildungslegende nichts anderes ergibt. Sofern das betreffende Material nicht unter der genannten Creative Commons Lizenz steht und die betreffende Handlung nicht nach gesetzlichen Vorschriften erlaubt ist, ist für die oben aufgeführten Weiterverwendungen des Materials die Einwilligung des jeweiligen Rechteinhabers einzuholen.

Weitere Details zur Lizenz entnehmen Sie bitte der Lizenzinformation auf http://creativecommons.org/licenses/by/4.0/deed.de.

\section{Literatur}

Allen M (2016) Strategic communications for sustainable organizations. Theory and practice. Springer, Cham

Bekmeier-Feuerhahn S, Bögel PM, Koch C (2017) Investigating internal CSR communication: building a theoretical framework. In: Diehl S, Karmasin M, Müller B, Terlutter R, Weder F (Hrsg) Handbook of integrated CSR communication. Springer, Cham, S 89-107

Berner W (2012) Culture Change: Unternehmenskultur als Wettbewerbsvorteil. Schäffer-Poeschel, Stuttgart

Bolton SC, Kim RC, O'Gorman KD (2011) Corporate social responsibility as a dynamic internal organizational process: a case study. J Bus Ethics 101(1):61-74

Brosius HB, Haas A, Koschel F (2016) Methoden der empirischen Kommunikationsforschung: Eine Einführung. Springer, Wiesbaden

Brugger F (2010) Nachhaltigkeit in der Unternehmenskommunikation: Bedeutung, Charakteristika und Herausforderungen. Springer, Wiesbaden

Buchholz U, Knorre S (2019) Change Management. In: Buchholz U, Knorre S (Hrsg) Interne Kommunikation und Unternehmensführung: Theorie und Praxis eines kommunikationszentrierten Managements. Springer, Wiesbaden, S 239-260

Buchholz U, Schach A, von der Haar V (2019) Werte und Metaphern in der Unternehmenskommunikation: Sensemaking, Mindset und Sprachen. Springer VS, Wiesbaden

Carollo L, Guerci M (2017) Between continuity and change: CSR managers' occupational rhetorics. J Organ Chang Manag 30(4): 632-646. https://doi.org/10.1108/JOCM-05-2016-0073

Cramer J, van der Heijden A, Jonker J (2006) Corporate social responsibility: making sense through thinking and acting. Bus Ethics 15(4):380-389. https://doi.org/10.1111/j.1467-8608.2006.00459. $\mathrm{X}$

Daft RL, Lengel RH (1986) Organizational information requirements, media richness and structural design. Manage Sci 32(5):554-571. https://doi.org/10.1287/mnsc.32.5.554

Deutinger G (2016) Mitten im Wandel. Die Rollen der internen KommunikatorInnen verändert sich derzeit radikal. In: Nowak R, Roither M (Hrsg) Interne Organisationskommunikation. Springer, Wiesbaden, S 93-107
Deutinger G (2017) Kommunikation im Change: erfolgreich kommunizieren in Veränderungsprozessen. Gabler, Berlin

Dolphin RL (2005) Internal communications. Today's strategic imperative. J Mark Commun 11(3):171-190. https://doi.org/10.1080/ 1352726042000315414

Dunphy D, Benn S, Griffiths A (2007) Organizational change for corporate sustainability: a guide for leaders and change agents of the future. Routledge, Milton Park, Abingdon, Oxon, NY

Engert S, Baumgartner RJ (2016) Corporate sustainability strategy-bridging the gap between formulation and implementation. J Clean Prod 113:822-834. https://doi.org/10.1016/j.jclepro. 2015.11.094

Fink S, Grupe M (2020) Innovations- und Technologiekommunikation: Komplexe Themen vermitteln und positionieren. In: Zerfaß A, Piwinger M, Röttger U (Hrsg) Handbuch Unternehmenskommunikation. Springer Gabler, Wiesbaden, S 1-19 https://doi.org/10. 1007/978-3-658-03894-6_45-1

Galtung J, Ruge MH (1965) The structure of foreign news. The presentation of the Conge, Cuba and Cyprus crises in four Norwegian newspapers. J Peace Res 2:64-91. https://doi.org/10.1177/ 002234336500200104

Gelbmann U, Baumgartner RJ (2012) Strategische Implementierung von CSR in KMU. In: Schneider A, Schmidpeter R (Hrsg) Corporate Social Responsibility. Springer, Berlin, Heidelberg, S 285-298

Gentile GC, Wetzel R, Wolf P (2015) The non-sense of organizational morality: Processing corporate social responsibility between formal and informal organizational structures. J Glob Responsib 6(1):19-44. https://doi.org/10.1108/JGR-11-2014-0030

Gläser J, Laudel G (2009) Experteninterviews und qualitative Inhaltsanalyse. VS, Wiesbaden

Göll E, Clausen J, Behrendet S, Kahlenborn W (2019) Auf dem Weg zu einer Green Economy: Wie die sozialökologische Transformation gelingen kann. transcript, Bielefeld

Hanke T, Stark W (2009) Strategy development: conceptual framework on corporate social responsibility. J Bus Ethics 85:507-516

Helfferich C (2019) Leifaden- und Experteninterviews. In: Baur N, Blasius J (Hrsg) Handbuch Methoden der empirischen Sozialforschung. Springer, Wiesbaden, S 669-689

Huck-Sandhu S (2013) Orientierung von Mitarbeitern - ein mikrotheoretischer Ansatz für die interne Kommunikation. In: Zerfaß A, Rademacher L, Wehmeier S (Hrsg) Organisationskommunikation und Public Relations. Forschungsparadigmen und neue Perspektiven. Springer VS, Wiesbaden, S 223-255

Huck-Sandhu S (2014) Corporate Messages entwickeln und steuern. Agenda Setting, Framing, Storytelling. In: Zerfaß A, Piwinger M (Hrsg) Handbuch Unternehmenskommunikation: Strategie, Management, Wertschöpfung. Springer Gabler, Wiesbaden, S 651-668

Huck-Sandhu S (2020) Mitarbeiterkommunikation aus Sicht der PRForschung. In: Einwiller S, Sackmann S, Zerfaß A (Hrsg) Handbuch Mitarbeiterkommunikation: Interne Kommunikation in Unternehmen. Springer Gabler, Wiesbaden, S 1-17

Kaiser R (2014) Qualitative Experteninterviews: Konzeptionelle Grundlagen und praktische Durchführung. Springer, Wiesbaden

Karst K, Segler T, Gruber KF (2000) Unternehmensstrategien erfolgreich umsetzen durch Commitment Management. Springer, Berlin, Heidelberg

Kaune A, Wagner AS (2016) Change Communication: Die Rede als Instrument im Kontext von Theorie, Empirie und Praxis. Springer, Wiesbaden

Kipping K, Nierstenhöfer A (2009) Mitarbeiterorientierung in Zeiten fundamentaler Veränderungen. In: Huck-Sandhu S (Hrsg) Unternehmenskommunikation in Zeiten der Wirtschaftskrise, S 141-164 (Universität Hohenheim, Lehrstuhl für Kommunikationswissenschaft und Journalistik) 
Klasen J (2019) Business Transformation: Praxisorientierter Leitfaden zur erfolgreichen Neuausrichtung von Unternehmen und Geschäftsfeldern. Springer Gabler, Wiesbaden

Kleinfeld A, Kettler A (2017) Wertemanagement und interne (CSR-) Kommunikation. In: Wagner R, Roschker N, Moutchnik A (Hrsg) CSR und interne Kommunikation: Forschungsansätze und Praxisbeiträge. Springer Gabler, Berlin, S 87-101

Kleinfeld A, Schnurr J (2010) CSR erfolgreich umsetzen. In: Hardtke A, Kleinfeld A (Hrsg) Gesellschaftliche Verantwortung von Unternehmen: Von Ideen der Corporate Social Responsibility zur erfolgreichen Umsetzung. Springer, Wiesbaden, S 286-359

Krüger W (2014) Das 3W-Modell: Bezugsrahmen für das Wandlungsmanagement. In: Krüger W, Bach N (Hrsg) Excellence in Change: Wege zur strategischen Erneuerung. Springer Gabler, Wiesbaden, S $1-32$

Lauer T (2010) Change Management: Grundlagen und Erfolgsfaktoren. Springer Gabler, Heidelberg

Lauer T (2019) Change Management: Grundlagen und Erfolgsfaktoren, 3. Aufl. Springer Gabler, Heidelberg

Lünenborg M (2005) Journalismus als kultureller Prozess. Zur Bedeutung von Journalismus in der Mediengesellschaft. Ein Entwurf. VS, Wiesbaden

Maon F, Lindgreen A, Swaen V (2009) Designing and implementing corporate social responsibility: an integrative framework grounded in theory and practice. J Bus Ethics 87(1):71-89. https://doi. org/10.1007/s10551-008-9804-2

Mast C (2008) Change Communication zwischen Gefühl und Kalkül: Theoretische Überlegungen und Ergebnisse aus Umfragen (Universität Hohenheim, Lehrstuhl für Kommunikationswissenschaft und Journalistik)

Mast C (2016) Unternehmenskommunikation: ein Leitfaden, 6. Aufl. UVK-Verlagsgesellschaft mbH, UVK Lucius, Konstanz, München

Mast C (2019) Unternehmenskommunikation: ein Leitfaden, 7. Aufl. UVK-Verlagsgesellschaft mbH, München

Mast C, Fiedler K (2007) Nachhaltige Unternehmenskommunikation. In: Michelsen G, Godemann J (Hrsg) Handbuch Nachhaltigkeitskommunikation: Grundlagen und Praxis. oekom, München, S 567-578

Mast C, Huck S (2008) Internal communication and leadership. In: Zerfaß A, van Ruler B, Sriramesh K (Hrsg) Public relations research: European and international perspectives and innovations. VS, Wiesbaden, S 147-162

Mayring P (2010) Qualitative Inhaltsanalyse: Grundlagen und Techniken. Beltz, Weinheim, Basel

Merten K (1999) Grundlagen, 3. Aufl. Einführung in die Kommunikationswissenschaft, Bd. I. LIT, Hamburg

Mey G, Mruck K (2011) Qualitative Interviews. In: Naderer G, Balz E (Hrsg) Marktforschung in Theorie und Praxis: Grundlagen - Methoden - Anwendungen. Gabler, Wiesbaden, S 259-288

Prexl A (2010) Nachhaltigkeit kommunizieren - nachhaltig kommunizieren: Analyse des Potenzials der Public Relations für eine nachhaltige Unternehmens- und Gesellschaftsentwicklung. VS, Wiesbaden

Quirke B (2008) Making the connections: using internal communications to turn strategy into action. Routledge, Milton Park

Rank S, Scheinpflug R (2010) Change Management in der Praxis: Beispiele, Methoden, Instrumente. Schmidt, Berlin

Raps A (2017) Erfolgsfaktoren der Strategieimplementierung: Konzeption, Instrumente und Fallbeispiele. Springer Gabler, Wiesbaden

Reiners H (2015) Change-Kommunikation ist vor allem StrategieKommunikation: Also Chefsache. In: Iskan S, Staudt E (Hrsg) Strategic Change. Springer, Wiesbaden, S 91-110
Röttger U (2009) Campaigns (f)or a better world? In: Röttger U (Hrsg) PR-Kampagnen. Über die Inszenierung von Öffentlichkeit, 4. Aufl. VS, Wiesbaden, S 9-23

Sackmann S (2019) Die Bedeutung der Unternehmenskultur für die Mitarbeiterkommunikation und ihre wechselseitige Beeinflussung. In: Einwiller S, Sackmann S, Zerfaß A (Hrsg) Handbuch der Mitarbeiterkommunikation. Springer, Wiesbaden, S 1-19

Sandelowski M (1995) Sample size in qualitative research. Res Nurs Health 18(2):179-183. https://doi.org/10.1002/nur.4770180211

Sawczyn-Müller A, Krohn C (2017) Glaubwürdigkeit und Transparenz in der CSR-Kommunikation. In: Wagner R, Roschker N, Moutchnik A (Hrsg) CSR und Interne Kommunikation: Forschungsansätze und Praxisbeiträge. Springer Gabler, Berlin, S 1-19

Schaltegger S, Herzig C, Kleiber O, Klinke T, Müller J (2007) Nachhaltigkeitsmanagement in Unternehmen: von der Idee zur Praxis: Managementansätze zur Umsetzung von Corporate Social Responsibility und Corporate Sustainability. Centre for Sustainability Management (CSM)/Bundesministerium für Umwelt, Naturschutz und Reaktorsicherheit (BMU)/econsense - Forum Nachhaltige Entwicklung der Deutschen Wirtschaft e. V., Lüneburg

Schultz F, Wehmeier S (2011) Zwischen Struktur und Akteur: Organisationssoziologische und -theoretische Perspektiven auf Corporate Social Responsibility. In: Raupp J, Jarolimek S, Schultz F (Hrsg) Handbuch CSR: Kommunikationswissenschaftliche Grundlagen, disziplinäre Zugänge und methodische Herausforderungen. Mit Glossar. VS, Wiesbaden, S 372-392

Schulz W (1990) Die Konstruktion von Realität in den Nachrichtenmedien. Analyse der aktuellen Berichterstattung. Alber, Freiburg, München

Spachmann K, Huck-Sandhu S (2020) Von der Mitarbeiterinformation zu Orientierung und Enabling. PR-Magazin 3:68-78

Steffen A, Doppler S (2019) Einführung in die qualitative Marktforschung: Design - Datengewinnung - Datenauswertung. Springer, Wiesbaden

Wagner R (2019) Effektive interne CSR-Kommunikation: Sinn stiften und motivieren für eine nachhaltige Unternehmensentwicklung. Springer, Wiesbaden

Walter BL (2017) Change Management und CSR-Kommunikation. In: Wagner R, Roschker N, Moutchnik A (Hrsg) CSR und Interne Kommunikation: Forschungsansätze und Praxisbeiträge. Springer Gabler, Berlin, S 103-114

Weder F, Karmasin M (2015) CSR - Potenziale für die PR-Forschung. In: Fröhlich R, Szyszka P, Bentele G (Hrsg) Handbuch der Public Relations. Springer, Wiesbaden, S 933-948

Weick KE (1985) Der Prozess des Organisierens. Suhrkamp, Frankfurt a.M.

Weick KE (1995) Sensemaking in organizations. SAGE, Thousand Oaks

Wieland J (2004) Wozu Wertemanagement? Ein Leitfaden für die Praxis. In: Wieland J (Hrsg) Handbuch Wertemanagement. Murmann, Hamburg, S 13-52

Zahn E, Goll F, Reichel A (2008) Organisationales Sensemaking und nachhaltigkeitsorientierter Wettbewerb. In: Gronau N (Hrsg) Wettbewerbsfähigkeit durch Arbeits- und Betriebsorganisation. GITO, Berlin, S 21-38

Zerfaß A, Huck-Sandhu S (2007) Innovationskommunikation: Neue Produkte, Ideen und Technologien erfolgreich positionieren. In: Piwinger M, Zerfaß A (Hrsg) Handbuch Unternehmenskommunikation. Gabler, Wiesbaden, S 847-858

Zerfaß A, Vercic D, Verhoeven P, Moreno Á, Tench R (2019) European communication monitor. Quadriga Media, Berlin, Brüssel

Zowislo-Grünewald N, Schwab H (2003) Interne Kommunikation im Veränderungsprozess: Mitarbeiter gezielt informieren und erfolgreich einsetzen. Gabler, Wiesbaden 